\title{
WEAKLY REGULAR AND SELF-INJECTIVE LEAVITT PATH ALGEBRAS OVER ARBITRARY GRAPHS
}

\author{
G. ARANDA PINO, K. M. RANGASWAMY, AND M. SILES MOLINA
}

\begin{abstract}
We characterize the Leavitt path algebras over arbitrary graphs which are weakly regular rings as well as those which are self-injective. In order to reach our goals we extend and prove several results on projective, injective and flat modules over Leavitt path algebras and, more generally, over (not necessarily unital) rings with local units.
\end{abstract}

\section{INTRODUCTION AND PRELIMINARIES}

For a graph $E$ and field $K$, the Leavitt path algebras $L_{K}(E)$ can be regarded as both a broad generalization of the algebras constructed by W. G. Leavitt in [38] to produce rings that do not satisfy the IBN property, and as the algebraic siblings of the graph $\mathrm{C}^{*}$-algebras $C^{*}(E)$, which in turn are the analytic counterpart and descendant from the algebras investigated by J. Cuntz in [27].

The first appearance of $L_{K}(E)$ took place in the papers [3] and [17], in the context of row-finite graphs (countable graphs such that every vertex emits only a finite number of edges). Although their history is very recent, a flurry of activity has followed since the beginning of the theory, in several different directions: characterization of algebraic properties of $L_{K}(E)$ in terms of graph properties of $E$ (see for instance [3, 4, 6, 18, 23]); study of the modules over $L_{K}(E)$ in [14, 21] among others; computation of various substructures such as the Jacobson radical, the center, the socle and the singular ideal in [5, 19, 21, 42] respectively; investigation of the relationship and connections with $C^{*}(E)$ and general $\mathrm{C}^{*}$-algebras $[9,17$, 20, 24]; classification programs [1, 2, 10]; K-Theory [14, 15, 17]; and generalization to arbitrary graphs, which has been done in two stages, first for countable but not necessarily row-finite graphs in $[5,22,43]$, and then for completely arbitrary graphs in $[7,8,32]$.

The present paper tackles several of these approaches altogether. We focus on the characterization of algebraic-theoretic properties of $L_{K}(E)$ in terms of graph-theoretic properties of $E$ : the main objective is to keep on exploring regularity conditions for $L_{K}(E)$, thus extending the characterization of von Neumann regular Leavitt path algebras of [7]. Concretely, we completely determine the weakly regular and self-injective Leavitt path algebras in Theorem 3.15 and Theorem 4.7 respectively.

Further, along the way we also handle the study of modules over $L_{K}(E)$ (needed in order to get our main theorems) as well as the arbitrary graphs problem, as we actually prove our results in the most general context, that is, for not necessarily row-finite and not necessarily countable graphs. Finally, we hope and foresee that the characterizations above, not having been considered for $C^{*}(E)$ yet, could in turn be useful tools and inspiration in the theory of graph $\mathrm{C}^{*}$-algebras.

2000 Mathematics Subject Classification. Primary 16D70.

Key words and phrases. Leavitt path algebra, weakly regular, self-injective, arbitrary graph. 
The article is organized as follows. We devote the first section to the basics on Leavitt path algebras and to fix the modules (over non-unital rings) we will consider: those which are unital and nondegenerate (the definition can be found later). Section 2 addresses to projective, injective and flat modules over non-unital rings. We use the homological notions, borrowed from the unital case. Neither all the results for unital rings remain valid for arbitrary rings, nor the case of Leavitt path algebras is translated word by word from the case of rings with local units (used as they provide a more general setting than Leavitt path algebras). While $R$ as a left $R$-module is not necessarily projective, for $R$ a non-unital ring (see [13]), this is not the case for Leavitt path algebras. The projective modules over a ring $R$ are precisely the projective modules over the unitization $R^{1}$ of $R$ (definition recalled later), as proved in Proposition 2.4. This does not happen for injective modules (see Example 2.7), contrary to Faith's statement in [29, Pg. 11]. We close Section 2 with the characterization of semisimple Leavitt path algebras in terms of its projective and injective modules (Proposition 2.27).

The description of weakly regular Leavitt path algebras is the main concern of Section 3. Right (left) weakly regularity, also called right (left) fully idempotency, was introduced by Ramamurthi in [40] (a ring $R$ is right weakly regular if $I^{2}=I$ for every right ideal $I$ ).

We start the section by characterizing right (left) weakly regular rings in the case of rings with local units (Theorem 3.1) and follow by showing that right (left) weakly regularity is a Morita invariant property for rings with local units (Theorem 3.13). The main result of the section (Theorem 3.15) characterizes weakly regular Leavitt path algebras in terms of ring properties, of properties of the graph, and of the lattice of its ideals. Concretely, we show that the weakly regular Leavitt path algebras are precisely the exchange Leavitt path algebras, equivalently, those whose graphs satisfy Condition (K), and they coincide with the Leavitt path algebras such that every ideal is graded. We also show that any graded ideal in a Leavitt path algebra can again be realized as a Leavitt path algebra, and this is useful in showing that a graph $E$ satisfies Condition $(K)$ if and only if every ideal in the Leavitt path algebra $L_{K}(E)$ has local units.

In the last section of the paper, we study and characterize self-injective Leavitt path algebras. They are precisely the semisimple ones (see Theorem 4.7), that is, those whose graph is row-finite, acyclic and every infinite path contains a line point (i.e., a vertex which is not a bifurcation and such that there are no cycles based at it).

First we collect various notions concerning graphs, after which we define Leavitt path algebras.

Definitions 1.1. A (directed) graph $E=\left(E^{0}, E^{1}, r, s\right)$ consists of two sets $E^{0}$ and $E^{1}$ together with maps $r, s: E^{1} \rightarrow E^{0}$. The elements of $E^{0}$ are called vertices and the elements of $E^{1}$ edges. For $e \in E^{1}$, the vertices $s(e)$ and $r(e)$ are called the source and range of $e$, respectively, and $e$ is said to be an edge from $s(e)$ to $r(e)$, represented by an arrow $s(e) \rightarrow r(e)$ when $E$ is drawn. If $s^{-1}(v)$ is a finite set for every $v \in E^{0}$, then the graph is called row-finite. If $E^{0}$ is finite and $E$ is row-finite, then $E^{1}$ must necessarily be finite as well; in this case we say simply that $E$ is finite.

A vertex which emits no edges is called a sink. A vertex $v$ is called an infinite emitter if $s^{-1}(v)$ is an infinite set, and a regular vertex otherwise. The set of infinite emitters will be denoted by $E_{\text {inf }}^{0}$. A path $\mu$ in a graph $E$ is a finite sequence of edges $\mu=e_{1} \ldots e_{n}$ such that $r\left(e_{i}\right)=s\left(e_{i+1}\right)$ for $i=1, \ldots, n-1$. In this case, $s(\mu)=s\left(e_{1}\right)$ and $r(\mu)=r\left(e_{n}\right)$ are the source 
and range of $\mu$, respectively, and $n$ is the length of $\mu$. We also say that $\mu$ is a path from $s\left(e_{1}\right)$ to $r\left(e_{n}\right)$, and we denote by $\mu^{0}$ the set of its vertices, i.e., $\left\{s\left(e_{1}\right), r\left(e_{1}\right), \ldots, r\left(e_{n}\right)\right\}$. For any set $Y$ of paths, denote $r(Y):=\{r(\alpha) \mid \alpha \in Y\}$ and $s(Y):=\{s(\alpha) \mid \alpha \in Y\}$. We view the elements of $E^{0}$ as paths of length 0

If $\mu$ is a path in $E$, and if $v=s(\mu)=r(\mu)$, then $\mu$ is called a closed path based at $v$. If $s(\mu)=r(\mu)$ and $s\left(e_{i}\right) \neq s\left(e_{j}\right)$ for every $i \neq j$, then $\mu$ is called a cycle. A graph which contains no cycles is called acyclic.

An edge $e$ is an exit for a path $\mu=e_{1} \ldots e_{n}$ if there exists $i$ such that $s(e)=s\left(e_{i}\right)$ and $e \neq e_{i}$. We say that $E$ satisfies Condition (L) if every cycle in $E$ has an exit. Let $M$ be a subset of $E^{0}$. A path in $M$ is a path $\alpha$ in $E$ with $\alpha^{0} \subseteq M$. We say that a path $\alpha$ in $M$ has an exit in $M$ if there exists $e \in E^{1}$ which is an exit for $\alpha$ and such that $r(e) \in M$.

Recall that a closed simple path based at a vertex $v$ is a path $\mu=e_{1} \cdots e_{t}$ such that $s(\mu)=r(\mu)=v$ and $s\left(e_{i}\right) \neq v$ for all $2 \leq i \leq t$. We denote the set of closed simple paths based at $v$ by $C S P(v)$. Further, $E$ is said to satisfy Condition $(\mathrm{K})$ if for each vertex $v$ on a closed simple path there exist at least two distinct closed simple paths based at $v$.

We define a relation $\geq$ on $E^{0}$ by setting $v \geq w$ if there exists a path in $E$ from $v$ to $w$. A subset $H$ of $E^{0}$ is called hereditary if $v \geq w$ and $v \in H$ imply $w \in H$. A hereditary set is saturated if every regular vertex which feeds into $H$ and only into $H$ is again in $H$, that is, if $s^{-1}(v) \neq \emptyset$ is finite and $r\left(s^{-1}(v)\right) \subseteq H$ imply $v \in H$. Denote by $\mathcal{H}_{E}$ the set of hereditary saturated subsets of $E^{0}$.

We recall here some graph-theoretic constructions which will be of use. Let $E$ be a rowfinite graph. For a hereditary subset $H$ of $E^{0}$, the quotient graph $E / H$ is defined as

$$
\left(E^{0} \backslash H,\left\{e \in E^{1} \mid r(e) \notin H\right\},\left.r\right|_{(E / H)^{1}},\left.s\right|_{(E / H)^{1}}\right),
$$

and the restriction graph is

$$
E_{H}=\left(H,\left\{e \in E^{1} \mid s(e) \in H\right\},\left.r\right|_{\left(E_{H}\right)^{1}},\left.s\right|_{\left(E_{H}\right)^{1}}\right) .
$$

Throughout this section, $K$ will denote an arbitrary base field.

The Leavitt path $K$-algebra $L_{K}(E)$ is defined to be the $K$-algebra generated by the set $E^{0} \cup E^{1} \cup\left\{e^{*} \mid e \in E^{1}\right\}$ with the following relations:

(i) $v w=\delta_{v, w} v$ for all $v, w \in E^{0}$.

(ii) $s(e) e=\operatorname{er}(e)=e$ for all $e \in E^{1}$.

(iii) $r(e) e^{*}=e^{*} s(e)=e^{*}$ for all $e \in E^{1}$.

(iv) $e^{*} f=\delta_{e, f} r(e)$ for all $e, f \in E^{1}$.

(v) $v=\sum_{e \in s^{-1}(v)} e e^{*}$ for every $v \in E^{0}$ that is neither a sink nor an infinite emitter.

The elements of $E^{1}$ are called real edges, while for $e \in E^{1}$ we call $e^{*}$ a ghost edge. The set $\left\{e^{*} \mid e \in E^{1}\right\}$ will be denoted by $\left(E^{1}\right)^{*}$. We let $r\left(e^{*}\right)$ denote $s(e)$, and we let $s\left(e^{*}\right)$ denote $r(e)$. If $\mu=e_{1} \ldots e_{n}$ is a path in $E$, we write $\mu^{*}$ for the element $e_{n}^{*} \ldots e_{1}^{*}$ of $L_{K}(E)$. For any subset $H$ of $E^{0}$, we will denote by $I(H)$ the ideal of $L_{K}(E)$ generated by $H$. Note that if $E$ is a finite graph, then $L_{K}(E)$ is unital with $\sum_{v \in E^{0}} v=1_{L_{K}(E)}$; otherwise, $L_{K}(E)$ is a ring with a set of local units consisting of sums of distinct vertices.

For a ring $R$ the assertion that $R$ has local units means that each finite subset of $R$ is contained in a corner of $R$, that is, a subring of the form $\epsilon R \epsilon$ where $\epsilon$ is an idempotent of $R$. 
Note that since every Leavitt path algebra $L_{K}(E)$ has local units, it is the directed union of its corners.

The Leavitt path algebra $L_{K}(E)$ is a $\mathbb{Z}$-graded $K$-algebra, spanned as a $K$-vector space by $\left\{p q^{*} \mid p, q\right.$ are paths in $\left.E\right\}$. (Recall that the elements of $E^{0}$ are viewed as paths of length 0 , so that this set includes elements of the form $v$ with $v \in E^{0}$.) In particular, for each $n \in \mathbb{Z}$, the degree $n$ component $L_{K}(E)_{n}$ is spanned by elements of the form $\left\{p q^{*} \mid \operatorname{length}(p)-\operatorname{length}(q)=\right.$ $n\}$.

For any ring $R$ without identity, instead of dealing with the category of all left modules $R$-mod, we will work with the usual category $R$-Mod, which is the full subcategory of $R$-mod of the unital and nondegenerate left $R$-modules. Recall that an $R$-module $M$ is unital if $R M=M$ and it is nondegenerate if $R m=0$, with $m \in M$, implies $m=0$. We will also deal with the category Mod- $R$ of all unital and nondegenerate right modules over $R$.

Proposition 1.2. Let $R$ be a ring with local units and let $M$ be a unital left $R$-module. Then:

(i) $M$ is nondegenerate.

(ii) For every $m \in M$ there is a local unit $\epsilon \in R$ such that $\epsilon m=m$.

Proof.

(i). Assume $R m=0$ for some $m \in M$. Since $M$ is unital, we may write $m=\sum_{i=1}^{n} r_{i} m_{i}$ for some $r_{i} \in R$ and $m_{i} \in M$. Find $\epsilon \in R$, a local unit for all the $r_{i}$ 's. Then we have $m=\sum_{i=1}^{n} r_{i} m_{i}=\sum_{i=1}^{n} \epsilon r_{i} m_{i}=\epsilon\left(\sum_{i=1}^{n} r_{i} m_{i}\right)=\epsilon m \in R m=0$, that is, $m=0$ as needed.

(ii). Since $M$ is unital, given $m$ in $M$ there are elements $r_{1}, \ldots, r_{n}$ in $R$ and $m_{1}, \ldots, m_{n} \in M$ such that $m=r_{1} m_{1}+\cdots+r_{n} m_{n}$. Now $r_{1}, \ldots, r_{n} \in \epsilon R \epsilon$ for some local unit $\epsilon$ in $R$. Then $\epsilon m=m$.

\section{Projective, injective, free and flat modules}

In this section we outline some of the standard results on projective, injective and flat modules over rings which have local units. Many of these results have not been recorded, to the authors' knowledge, in any published literature and are needed in the subsequent sections when we deal with Leavitt path algebras.

As noted by Ánh and Márki in [13], a ring $R$ without identity may not be a projective $R$-module. In contrast, an easy argument (indicated below) shows that a Leavitt path algebra over an arbitrary graph is always projective as a module over itself. Other useful results on injective and flat modules over a ring with local units are also outlined.

Definition 2.1. A module $P$ in $R$-Mod is projective if for any epimorphism $B \stackrel{\eta}{\rightarrow} C \rightarrow 0$, with $B$ and $C$ in $R$-Mod, and every homomorphism $f: P \rightarrow C$ there exists a homomorphism $g: P \rightarrow B$ such that the following diagram is commutative.

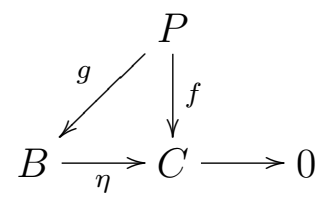

The following is a first example of projective $R$-module. 
Proposition 2.2. Let $R$ be any ring. Then for any idempotent $\epsilon \in R$, the left ideal $R \epsilon$ is a projective module in the category $R$-Mod.

Proof. Consider the following diagram with exact row in the category $R$-Mod.

\section{has to be $\mathrm{R}$}

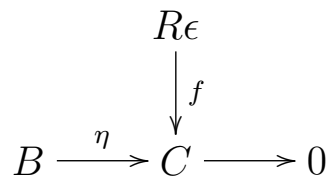

Observe that $\epsilon=\epsilon^{2} \in R \epsilon$. Let $f(\epsilon)=c$ and let $b^{\prime} \in B$ such that $\eta\left(b^{\prime}\right)=c$. Now $\epsilon c=\epsilon f(\epsilon)=f\left(\epsilon^{2}\right)=f(\epsilon)=c$ and so $\eta\left(\epsilon b^{\prime}\right)=\epsilon \eta\left(b^{\prime}\right)=\epsilon c=c$. Thus $\epsilon b^{\prime}-b^{\prime}=k \in$ ker $\eta$. From $\epsilon b^{\prime}=b^{\prime}+k$ we get (i) $\epsilon b^{\prime}=\epsilon b^{\prime}+\epsilon k$ which implies that $\epsilon k=0$ and (ii) $\epsilon\left(b^{\prime}+k\right)=\epsilon\left(\epsilon b^{\prime}\right)=$ $\epsilon b^{\prime}=b^{\prime}+k$. Then $b=b^{\prime}+k$ satisfies $\epsilon b=b$ and $\eta(b)=c$. Since $x \epsilon=0$ implies $x b=x \epsilon b=0$, the map $g: L_{K}(E) \rightarrow B$ given by $g(x \epsilon)=x b$ is a well-defined homomorphism that satisfies $\eta g=f$. Hence $\mathrm{L}_{K}(E) \epsilon$ is projective.

Corollary 2.3. For an arbitrary graph $E$ and field $K$ the Leavitt path algebra $L_{K}(E)$ is a projective module in the category $L_{K}(E)$-Mod.

Proof. Note that $L_{K}(E)=\oplus_{v \in E^{0}} L_{K}(E) v$. Since $L_{K}(E) v$ is projective (by Proposition 2.2) and the direct sum of projective modules in $L_{K}(E)$-Mod is again projective, we conclude that $L_{K}(E)$ is a projective module in $L_{K}(E)$-Mod.

Although, as it has been said before, a ring $R$ without identity need not be a projective $R$ module, one way to consider finitely generated projective modules over such a ring is indicated in [17, pg. 163] as follows: a finitely generated left $R$-module $P$ is defined as projective if, as a left module over the unitization $R^{1}, P$ is projective (recall that for a ring $R$ the unitization of $R$ is defined as $R^{1}:=R \oplus \mathbb{Z}$ with addition componentwise and multiplication defined by $(x, n)(y, m)=(x y+n y+m x, n m)$ for all $x, y \in R$ and $n, m \in \mathbb{Z}$; note that $R$ can be seen as an ideal of $R^{1}$ by identifying it to $\{(x, 0)$ with $\left.x \in R\}\right)$.

The following Proposition shows that for a ring $R$ with local units this new definition coincides with the usual definition of projective modules over $R$ (Definition 2.1).

Proposition 2.4. Let $R$ be a ring with local units and let $P \in R$-Mod. Then:

(i) For any left $R^{1}$-module $B, \operatorname{Hom}_{R}(P, B)=\operatorname{Hom}_{R^{1}}(P, B)$.

(ii) $P$ is projective as an $R$-module if and only $P$ is projective as an $R^{1}$-module (with the definition of projectivity given in Definition 2.1).

Proof. (i). Let $g: P \rightarrow B$ be an $R$-homomorphism. Consider $x \in P$. By Proposition 1.2 (ii), there is a local unit $v \in R$ such that $v x=x$. Then, for any $s \in R^{1}$ we have $g(s x)=g(s v x)=s v g(x)$, as $s v \in R$ and $g$ is an $R$-morphism. But $s v g(x)=s g(v x)=s g(x)$. Thus, $g$ is an $R^{1}$-homomorphism.

(ii). We need only to prove the "only if" part. Suppose $P$ is a projective $R$-module. Consider the following diagram of $R^{1}$-modules and $R^{1}$-morphisms with an exact row:

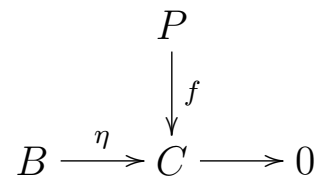


By projectivity, there is an $R$-morphism $g: P \rightarrow B$ such that $\eta g=f$. By Part (i) above, $g$ is an $R^{1}$-morphism, hence $P$ is a projective $R^{1}$-module.

We next consider the injective modules in $R$-Mod. As we shall see, the statement corresponding to Proposition 2.4 (ii) is false for injective modules. We first recall the Baer Criterion for injectivity in $R$-Mod. We don't include its proof because it is almost identical to the corresponding one for modules over unital rings (see [41, Theorem 3.30]).

Proposition 2.5. (Baer Criterion). Let $R$ be a ring with local units. A module $M \in R$-Mod is injective if and only if for any left ideal $I$ of $R$ and any $R$-homomorphism $f: I \rightarrow M$ there is an R-homomorphism $g: R \rightarrow M$ such that $g_{\left.\right|_{I}}=f$.

Remark 2.6. Let $R$ be a unital ring and $R^{1}$ its unitization. Every left $R$-module $M$ can be made a unital $R^{1}$-module under the operation $(r, m) x=r x+m x$. Moreover every homomorphism of left $R$-modules $f: M \rightarrow N$ is an $R^{1}$-homomorphism, since for any $(r, m) \in R^{1}$ and $x \in M$, we have $f((r, m) x)=f(r x+m x)=r f(x)+m f(x)=(a, m) f(x)$. Thus $\operatorname{Hom}_{R}(M, N)=\operatorname{Hom}_{R^{1}}(M, N)$ for any left $R$-module $N$. On Page 11 of his book [29], Carl Faith deduces that this implies that a left $R$-module $M$ is injective if and only if $M$ is injective as a left $R^{1}$-module. This is false, as the following example shows.

Example 2.7. Let $\mathbb{Q}$ be the field of rational numbers and let $R$ be the ring consisting on the direct sum of countably infinite copies of $\mathbb{Q}$, say, $R=\oplus_{i=1}^{\infty} R_{i}$, with $R_{i} \cong \mathbb{Q}$. Clearly, $R$ is a non-unital ring. Since $R$ is a direct sum of simple $R$-modules, every $R$-module $M$ satisfying $R M=M$ and, in particular $R$, is injective as a left $R$-module. Let $R^{1}$ be the unitization of $R$. Then $R$ is not injective as an $R^{1}$-module. This is because $R$ is an essential right ideal in $R^{1}$ : if $(r, n) \in R^{1}$ is non-zero, then we can find a suitable element $(s, 0) \in R$ such that $(r, n) \cdot(s, 0)=(r s+n s, 0) \neq(0,0)$. So, if $R$ were injective, then $R=R^{1}$ would be a ring with identity ( as an injective $R^{1}$-module has no proper essential extensions). This is a contradiction, since, by construction, $R$ is a non-unital ring. Mod-R (Change

We next consider some preliminary results on flat modyles over rings with locar units.

Definition 2.8. Let $R$ be an arbitrary ring. We wik say that a module $M \in R$-Mod is flat if the functor $-\otimes_{R} M$ is exact on the categor $R$-Mod. In other words, if whenever $0 \rightarrow A \rightarrow B \rightarrow C \rightarrow 0$ is a short exact sequence ir $R$-Mod then

$$
0 \longrightarrow A \otimes_{R} M \longrightarrow B \otimes_{R} M \longrightarrow C \otimes_{R} M \longrightarrow 0
$$

is a short exact sequence. Since $-\otimes_{R} M$ is exact on the right hand side, the module $M$ is flat if any monomorphism $A \rightarrow B$ gives rise to a monomorphism $A \otimes_{R} M \rightarrow B \otimes_{R} M$. The notion of flat right $R$-module can be obtained from this one in the obvious way.

Lemma 2.9. Let $R$ be a ring with local units. For any $M \in$ Mod-R the map $\mu_{M}: M \otimes R \rightarrow M$ given by $\sum_{i=1}^{n} m_{i} \otimes r_{i} \mapsto \sum_{i=1}^{n} m_{i} r_{i}$ is an isomorphism of right $R$-modules.

Proof. Since $M R=M$, this map is clearly an epimorphism. Suppose $\sum_{i=1}^{n} m_{i} r_{i}=0$. Let $\epsilon$ be a local unit in $R$ satisfying $\epsilon r_{i}=r_{i} \epsilon=r_{i}$ for $i=1, \ldots, n$. Then $\sum_{i=1}^{n} m_{i} \otimes r_{i}=$ $\sum_{i=1}^{n} m_{i} \otimes r_{i} \epsilon=\sum_{i=1}^{n} m_{i} r_{i} \otimes \epsilon=\left(\sum_{i=1}^{n} m_{i} r_{i}\right) \otimes \epsilon=0 \otimes \epsilon=0$.

Corollary 2.10. A ring $R$ with local units is flat as a left $R$-module. 
Proof. In view of the above Lemma 2.9, the original proof for unital rings holds here (see [41, Proposition 3.46 (i)]). Indeed, let $0 \rightarrow A \stackrel{f}{\rightarrow} B$ be an exact sequence of right $R$-modules. Tensoring with the left $R$-module $R$, we get a commutative diagram

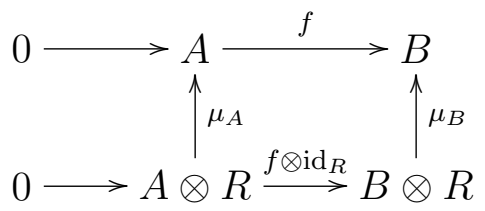

where $\mathrm{id}_{R}$ is the identity map on $R$ and $\mu_{A}, \mu_{B}$ are the isomorphisms defined in Lemma 2.9. Since $\mu_{A}, f$ and $\mu_{B}$ are all monomorphisms, so is $f \otimes \mathrm{id}_{R}$. Hence $R$ is flat in $R$-Mod.

More examples of projective and flat modules over rings with local units are given by the free modules we define in what follows.

Definition 2.11. Let $R$ be a ring with local units, and $F$ a left module in $R$-Mod. Suppose that there exist and index set $\mathcal{B}=\left\{b_{i}\right\}_{i \in \Lambda} \subseteq F$ and a set of idempotents $\mathcal{U}:=\left\{u_{i}\right\}_{i \in \Lambda} \subseteq R$ with $b_{i}=u_{i} b_{i}$ for every $i \in \Lambda$ satisfying: for every $x \in F$ there exists a unique family $\left\{r_{i}\right\}_{i \in \Lambda} \subseteq R$ with $r_{i}=r_{i} u_{i}$ for every $i \in \Lambda$, almost all $r_{i}=0$, such that $x=\sum_{i \in \Lambda} r_{i} b_{i}$. Then we say that $F$ is a $\mathcal{U}$-free left $R$-module, and $\mathcal{B}$ is called a $\mathcal{U}$-basis of $F$. Note that, in particular, $F=\bigoplus_{i \in \Lambda} R b_{i}$.

If $R$ is a ring with a unit element 1 then taking $u_{i}=1$ in Definition 2.11 we have the definition of free left $R$-module. On the other hand, Definition 2.11 can be considered as an extension of the notion of projective basis (see [41, Definition in pg. 106]).

Example 2.12. Every Leavitt path algebra $L_{K}(E)$ is an $E^{0}$-free left $L_{K}(E)$-module with basis $E^{0}$.

Remark 2.13. If $F$ is a $\mathcal{U}$-free left $R$-module, for $R$ a ring with local units, then (by the definition) $F$ is unital, and by Proposition 1.2 (i) $F \in R$-Mod, hence whenever we consider a free module, there is no need of specifying that it is a unital nondegenerate left $R$-module.

The first property a $\mathcal{U}$-basis has is that every homomorphism of a $\mathcal{U}$-free module is determined by the image of the elements of the $\mathcal{U}$-basis.

Lemma 2.14. Let $R$ be a ring with local units and $F$ a $\mathcal{U}$-free left $R$-module with basis $\mathcal{B}=\left\{b_{i}\right\}_{i \in \Lambda}$. Consider a family $\left\{a_{i}\right\}_{i \in \Lambda}$ of elements in a left $R$-module $A$. Then there exists a unique homomorphism of left $R$-modules $f: F \rightarrow A$ such that $f\left(b_{i}\right)=u_{i} a_{i}$ for every $i \in \Lambda$ (where $\left.\mathcal{U}=\left\{u_{i}\right\}_{i \in \Lambda}\right)$.

Proof. For any $x \in F$, let $\left\{r_{i}\right\}_{i \in \Lambda} \subseteq R$ be the unique family such that $r_{i}=r_{i} u_{i}$ for every $i \in \Lambda$, almost all $r_{i}=0$, and $x=\sum_{i \in \Lambda} r_{i} b_{i}$. Define $f: F \rightarrow A$ by $f(x):=\sum_{i \in \Lambda} r_{i} b_{i}$. The uniqueness of the $r_{i}$ 's implies that this map is well defined, and it is immediate to see that it is a homomorphism of left $R$-modules such that $f\left(b_{i}\right)=u_{i} a_{i}$ for every $i \in \Lambda$. It is also easy to check that $f$ is unique, as every element of $F$ is in the $R$-span of $\mathcal{B}$.

Lemma 2.15. Let $R$ be a ring with local units and $F$ a $\mathcal{U}$-free left $R$-module with basis $\mathcal{B}=\left\{b_{i}\right\}_{i \in \Lambda}$. Then $F$ is projective as a left $R$-module. 
Proof. Denote $\mathcal{U}=\left\{u_{i}\right\}_{i \in \Lambda}$, with the $u_{i}$ 's as in Definition 2.11. Consider the following diagram with exact row in the category $R$-Mod.

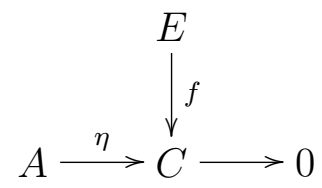

Surjectivity of $\eta$ implies that there exists a family $\left\{a_{i}\right\}_{i \in \Lambda} \subseteq A$ satisfying $\eta\left(a_{i}\right)=f\left(b_{i}\right)$ for every $i \in \Lambda$. By Lemma 2.14 there is a unique homomorphism $g: E \rightarrow A$ with $g\left(b_{i}\right)=u_{i} a_{i}$ for every $i \in \Lambda$. We claim that $\eta g=f$. Indeed, consider $x \in E$ and write $x=\sum_{i \in \Lambda} r_{i} b_{i}$, with the $r_{i}$ 's as in Definition 2.11. Then $\eta g(x)=\eta g\left(\sum_{i \in \Lambda} r_{i} b_{i}\right)=\eta\left(\sum_{i \in \Lambda} r_{i} g\left(b_{i}\right)\right)=\eta\left(\sum_{i \in \Lambda} r_{i} u_{i} a_{i}\right)=$ $\eta\left(\sum_{i \in \Lambda} r_{i} a_{i}\right)=\sum_{i \in \Lambda} r_{i} \eta\left(a_{i}\right)=\sum_{i \in \Lambda} r_{i} f\left(b_{i}\right)=f\left(\sum_{i \in \Lambda} r_{i} b_{i}\right)=f(x)$. This concludes the proof.

Remark 2.16. Example 2.12 and Lemma 2.15 imply that every Leavitt path algebra is projective as a left module over itself, result that we have obtained in Corollary 2.3.

As in the case of unital rings, arbitrary modules over a ring with local units can be described in terms of free $\mathcal{U}$-modules.

Proposition 2.17. Every module over a ring $R$ with local units is the epimorphic image of a $\mathcal{U}$-free module.

Proof. Let $M$ be in $R$-Mod. Consider $m \in M$; by Proposition 1.2 (ii) there exists a local unit $\epsilon_{m} \in R$ such that $\epsilon_{m} m=m$. This implies $M=\sum_{m \in M} R m=\sum_{m \in M} R \epsilon_{m} m$ and therefore the map $\oplus_{m \in M} R \epsilon_{m} \rightarrow M$ given by $\left(r_{m} \epsilon_{m}\right) \mapsto \sum_{m} r_{m} \epsilon_{m} m=\sum_{m} r_{m} m$ yields an epimorphism between $\oplus_{m \in M} R \epsilon_{m}$ and $M$. Moreover, $\oplus_{m \in M} R \epsilon_{m}$ is a $\mathcal{U}$-free left module for $\mathcal{U}:=\left\{\epsilon_{m}\right\}_{m \in M}$.

Proposition 2.18. Let $R$ be a ring with local units and $P \in R$-Mod. Then $P$ is projective if and only if every short exact sequence $0 \longrightarrow A \stackrel{f}{\longrightarrow} B \stackrel{g}{\longrightarrow} P \longrightarrow 0$ splits.

Proof. Follow the proof of the unital case ([41, Proposition 3.3]) and use Lemma 2.15 and Proposition 2.17.

The next result provides with a characterization of projective modules over rings with local units.

Theorem 2.19. Let $R$ a be a ring with local units and let $P$ be in $R$-Mod. Then $P$ is projective if and only if it is a direct summand of a $\mathcal{U}$-free let $R$-module.

Proof. Again, follow the unital case ([41, Theorem 3.5 (i)]) and use Propositions 2.17 and 2.18 and Lemma 2.15 .

Lemma 2.20. Let $R$ be a ring with local units. Then every $\mathcal{U}$-free left $R$-module is flat.

Proof. Consider a $\mathcal{U}$-free left $R$-module $F$. Let $\mathcal{U}:=\left\{u_{i}\right\}_{i \in \Lambda}$ and $\mathcal{B}=\left\{b_{i}\right\}_{i \in \Lambda} \subseteq F$ be as in Definition 2.11. Plainly, $R u_{i}$ is isomorphic, as a left $R$-module, to $R b_{i}=R u_{i} b_{i}$. Now, use the following facts: $R$ is flat (Corollary 2.10), $R \epsilon$ is a direct summand of $R$ for every idempotent $\epsilon \in R$, and flatness is inherited by direct summands, to get that every $R b_{i}$ is flat. Apply again that flatness behaves well with direct sums to obtain $F=\oplus_{i \in \Lambda} R b_{i}$ is flat. 
Corollary 2.21. Let $R$ be a ring with local units. Then every projective left $R$-module is flat.

Proof. Let $P$ be a projective left $R$-module. By Theorem 2.19 $P$ is a direct summand of a $\mathcal{U}$-free module $F$. Now, apply Lemma 2.20 and that every direct summand of a flat module is flat to get the result.

Corollary 2.22. Let $E$ be an arbitrary graph and $K$ any field. Then every projective module $P \in L_{K}(E)$-Mod is flat.

Many of the known characterizations of flat modules carry over to flat modules in $R$-Mod, for $R$ a ring with local units. We single out one proved by O. Villamayor for rings with identity (see [41, Theorem 3.62]).

First we need the following result, which can be proved going along the proof of $[41$, Proposition 3.60]. As a corollary we will obtain more examples of flat modules.

Proposition 2.23. Let $R$ be a ring with local units, $F$ a flat left $R$-module and $K$ a submodule of $F$. Then $F / K$ is a flat left $R$-module if and only if $K \cap I F=I K$ for every finitely generated right ideal $I$ of $R$.

Recall that for a semiprime ring $R$ the socle of $R$, denoted by $\operatorname{Soc}(R)$ is defined as the sum of all minimal left (or right) ideals of $R$. If there are no minimal one-sided ideals then $\operatorname{Soc}(R):=0$. A complete study of the socle of a Leavitt path algebra has been reached with $[?, 22,8]$.

Corollary 2.24. Let $R$ be a semiprime ring with local units. Then $R / \operatorname{Soc}(R)$ is flat.

Proof. It is known that the socle of a semiprime ring, which is an ideal of the ring, is von Neumann regular: for unital rings, see [41, Corollary 4.10]; for non-unital rings, apply Litoff's Theorem (see, for example [26, Theorem 4.3.11]) and the unital case. Hence, if we denote $\operatorname{Soc}(R)$ by $S$ we have $S \cap I R=I S$ for every finitely generated right ideal of $R$. This implies that $R / \operatorname{Soc}(R)$ is flat as a left $R$-module, by Proposition 2.23.

A similar argument shows that $R / \operatorname{Soc}(R)$ is flat as a right $R$-module.

Theorem 2.25. Let $R$ be a ring with local units and let $F$ be a $\mathcal{U}$-free left $R$-module. Then, for any submodule $S$ of $F$, the following conditions are equivalent:

(i) $F / S$ is a flat $R$-module.

(ii) For each element $x$ in $S$, there is a homomorphism $f: F \rightarrow S$ such that $f(x)=x$.

(iii) For each finite set of elements $\left\{x_{1}, \ldots, x_{n}\right\}$ of $S$, there is a homomorphism $f: F \rightarrow S$ such that $f\left(x_{i}\right)=x_{i}$ for all $i=1, \ldots, n$.

Proof. All we need is to repeat the original proof of Villamayor's Theorem for rings with identity (see [41, Theorem 3.62]) after replacing [41, Proposition 3.60] by Proposition 2.23 and [41, Lemma 3.61] by its counterpart for rings with local units, which remains valid.

We close the section by characterizing semisimple Leavitt path algebras in terms of its projective and injective modules. First, we need to prove that every Leavitt path algebra contains maximal one sided ideals. 
In general, a ring without identity need not have a maximal one sided ideal. This happens if $R$ is a ring whose additive part $R^{+}$is a torsion divisible abelian group. For example, let $R^{+}=\mathbb{Q} / \mathbb{Z}$, the additive group of rational numbers modulo the integers. First notice that the ring multiplication in $R$ is trivial, that is, $a \cdot b=0$ for any two elements $a, b \in R$. To see this, suppose $b$ is an element of additive order $n$. Since $R^{+}$is divisible, we can write $a=n c$ for some element $c$ of $R^{+}$. Then $a \cdot b=n c \cdot b=c \cdot(n b)=c \cdot 0=0$. Thus every subgroup of $R^{+}$is indeed an ideal of the (commutative) ring $R$. Being divisible, $R^{+}$has no maximal subgroups (see [30, Exercise 1, pg. 99]) and so the ring $R$ has no maximal one-sided ideals.

However, the ring $L_{K}(E)$ has maximal one-sided ideals as indicated below.

Lemma 2.26. For any graph $E$, the ring $L_{K}(E)$ has maximal left and right ideals.

Proof. Write $L_{K}(E)=\oplus_{v \in E^{0}} L_{K}(E) v$. For a fixed vertex $u$, the set

$$
\left\{I \mid I \text { a left ideal of } L_{K}(E), u \notin I \subsetneq L_{K}(E) u\right\}
$$

is inductive under the set inclusion and so, by Zorn's Lemma, there is a maximal member $M$. It is clear that, as a left $L_{K}(E)$-module, $M$ is a maximal submodule of $L_{K}(E) u$. Then $M \oplus\left(\oplus_{v \in E^{0} \backslash\{u\}} L_{K}(E) v\right)$ is a maximal left ideal of $L_{K}(E)$. It is possible to find, in a similar way, maximal right ideals.

Proposition 2.27. Let $E$ be an arbitrary graph and $K$ any field. The following are equivalent conditions:

(i) $\operatorname{Hom}_{L_{K}(E)}\left(S, L_{K}(E)\right) \neq 0$ for any simple module $S$ in $L_{K}(E)$-Mod.

(ii) Every simple $L_{K}(E)$-module is projective in $L_{K}(E)$-Mod.

(iii) $L_{K}(E)=\operatorname{Soc}\left(L_{K}(E)\right)$.

(iv) Every left $L_{K}(E)$-module is injective.

(v) Every left $L_{K}(E)$-module is projective.

Proof. The proof of (iii) $\Leftrightarrow$ (iv) and (iii) $\Leftrightarrow$ (v) is similar to the analogue results for unital rings (see [37, Theorem 2.8] and [37, Theorem 2.9], respectively), so we omit it.

(i) $\Rightarrow$ (ii). Suppose $S$ is a simple left $L_{K}(E)$-module with $\operatorname{Hom}_{L_{K}(E)}\left(S, L_{K}(E)\right) \neq 0$, so that there is a non-zero homomorphism $f: S \rightarrow L_{K}(E)$. Now $f(S) \cong S$ is a simple left ideal of $L_{K}(E)$ and since $L_{K}(E)$ is semiprime, $f(S)$ is generated (as a left ideal) by an idempotent $\epsilon \in L_{K}(E)$, that is, $f(S)=L_{K}(E) \epsilon$. By Proposition 2.2, $f(S)$, and hence $S$, is a projective left $L_{K}(E)$-module.

(ii) $\Rightarrow$ (iii). Lemma 2.26 implies that there exists a maximal left ideal $M$ of $L_{K}(E)$, hence $L_{K}(E) / M$ is a simple left $L_{K}(E)$-module; by our hypothesis, $L_{K}(E) / M$ is projective, hence $L_{K}(E)=I \oplus M$, for $I$ a left ideal of $L_{K}(E)$. This implies $I \cong L_{K}(E) / M$ is a simple left ideal of $L_{K}(E)$, hence it is contained in $S:=\operatorname{Soc}\left(L_{K}(E)\right)$. We claim that $S=L_{K}(E)$.

Suppose $S \subsetneq L_{K}(E)=\oplus_{v \in E^{0}} L_{K}(E) v$. Since the image of $S$ under the coordinate projection $L_{K}(E) \rightarrow L_{K}(E) v$ is a sum of simple left ideals, we conclude $S=\oplus_{v \in E^{0}}\left(S \cap L_{K}(E) v\right)$. If $S \neq L_{K}(E)$, there exists $v$ for which $S \cap L_{K}(E) v \subsetneq L_{K}(E) v$. By Zorn's Lemma there exists a submodule $H$ of $L_{K}(E) v$ maximal with respect to the property of containing $S \cap L_{K}(E) v$. In fact, it is a maximal submodule of $L_{K}(E) v$, hence $L_{K}(E) v / H$ is a simple $L_{K}(E)$-module. By (ii), it is projective, and so $L_{K}(E) v=H \oplus C$, for $C$ a left $L_{K}(E)$-module. Then $C \cong$ $L_{K}(E) v / H$ is a simple left $L_{K}(E)$-module having zero intersection with $S$ (as it is contained 
in $L_{K}(E) v$ and its intersection with $H$ is zero). But this is a contradiction. Hence, $L_{K}(E)=$ $S=\operatorname{Soc}\left(L_{K}(E)\right)$.

(iii) $\Rightarrow$ (i). If $L_{K}(E)=\operatorname{Soc}\left(L_{K}(E)\right)$, every left ideal of $L_{K}(E)$ is a direct summand. Since every simple left $L_{K}(E)$-module $S$ is of the form $L_{K}(E) / M$ for some maximal left ideal $M$ of $L_{K}(E)$ which is a direct summand, we conclude that $S$ is isomorphic to a direct summand of $L_{K}(E)$ and hence $\operatorname{Hom}_{L_{K}(E)}\left(S, L_{K}(E)\right) \neq 0$.

Remark 2.28. It is well known that conditions (ii) to (v) in Proposition 2.27 are equivalent for rings with local units. However, condition (i) needs not imply any of the conditions (ii), (iii), (iv) or (v) for arbitrary rings, not even for rings with identity. For example, let $p$ be a prime integer and $R=\mathbb{Z} / p^{2} \mathbb{Z}$ the ring of integers modulo $p^{2}$. Then multiplication by $p$ is an $R$-homomorphism giving rise to the exact sequence $0 \rightarrow p R \rightarrow R \rightarrow p R \rightarrow 0$. Clearly $p R \cong R / p R$ is (up to an isomorphism) the only simple $R$-module and $\operatorname{Hom}_{R}(p R, R) \neq 0$. But $p R$ is neither projective nor injective and $R \neq \operatorname{Soc}(R)$.

\section{WEAKLY REGULAR LEAVITT PATH ALGEBRAS}

The notion of right weakly regular ring was introduced by Ramamurthi in [40]. It can be found in the literature also as right fully idempotent ring (see, for example [11]).

A (not necessarily unital) ring $R$ is said to be right weakly regular if every right ideal $I$ is idempotent, that is, $I^{2}=I$. Left right weakly regular means that every left ideal is idempotent, while the ring is called weakly regular if it is left and right weakly regular.

In this section we will characterize right weakly regular Leavitt path algebras and will prove that the notion is left-right symmetric in this context. In general, a right weakly regular ring need not be left weakly regular: the paper [11] is devoted to find such an example of this fact.

We start first by a characterization of right weakly regular rings. The equivalences (i) $\Leftrightarrow$ (ii) and (i) $\Leftrightarrow(\mathrm{v})$ were proved, respectively, in [40, Proposition 1 and Remark 3].

Theorem 3.1. Let $R$ be a ring with local units. The following are equivalent conditions:

(i) $R$ is right weakly regular.

(ii) For every element $a$ in $R$ there exists $x \in R a R$ such that $a=a x$.

(iii) For every ideal $I$ of $R$ the left $R$-module $R / I$ is flat.

(iv) For every element $a$ in $R$ the left $R$-module $R / R a R$ is flat.

(v) For every ideal $I$ of $R$ and for every right ideal $Y$ of $R, Y \cap I=Y I$.

Proof. (i) $\Rightarrow$ (ii). For $a \in R$, by the hypothesis $a R=a R a R$; since $R$ has local units, there exists an idempotent $e \in R$ such that $a=a e$, hence $a \in a R=a R a R$, so $a=a x$ for some $x \in R a R$.

(ii) $\Rightarrow$ (iii). Let $I$ be an ideal of $R$. By Corollary 2.10, $R$ is flat as a left $R$-module, hence in order to prove flatness of $R / I$ it is enough to show that if $Y$ is a right ideal of $R$ then $I \cap Y R=Y I$ and apply Proposition 2.23. The containment $Y I \subseteq I \cap Y R$ is obvious. Now, let $y \in I \cap Y R$. By (ii), there exists $x \in R y R$ such that $y=y x$, therefore, $y=y x \in Y R x \subseteq Y R R y R \subseteq Y I$.

(iii) $\Rightarrow$ (iv) is a tautology.

(iv) $\Rightarrow$ (ii). Given $a \in R$, the flatness of the left $R$-module $R / R a R$ implies (by Corollary 2.10 and Proposition 2.23) $R a R \cap Y R=Y R a R$ for every right ideal $Y$ of $R$. In particular, for $Y=a R$ we have $R a R \cap a R R=a R R a R$. Use that $R$ has local units to get $a \in R a R \cap a R R$, 
hence $a \in a R a R$, that is, there exists a finite number of elements $r_{i}, s_{i} \in R$ such that $a=\sum a r_{i} a s_{i}=a \sum r_{i} a s_{i}=a x$ for $x=\sum r_{i} a s_{i} \in R a R$.

(ii) $\Rightarrow(\mathrm{v})$. Consider an ideal $I$ of $R$ and a right ideal $Y$ of $R$. It is clear that $Y I \subseteq Y \cap I$. To show the other containment, consider $a \in Y \cap I$; by (ii) there exists $x \in R a R$ such that $a=a x$. Finally, $a x \in a R a R \subseteq Y R I R \subseteq Y I$.

$(\mathrm{v}) \Rightarrow(\mathrm{i})$. Let $I$ be a right ideal of $R$. By (v) and using that $R$ has local units we have $I=I \cap R I=I R I=I^{2}$, so $R$ is right weakly regular.

An application of Theorem 3.1 provides with examples of weakly regular rings: the von Neumann regular rings and the simple rings with local units.

The following are examples of right weakly regular Leavitt path algebras.

Examples 3.2. (i) For every $n \geq 2$, the Leavitt algebra $L(1, n)$ is right weakly regular.

(ii) The Leavitt path algebra associated to the following graph is right weakly regular, although it is neither simple nor von Neumann regular:

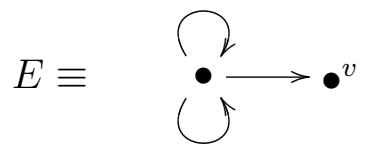

Proof. (i) follows because $L(1, n)$ is simple (see [3, Corollary 3.13 (iii)]).

(ii). As $E$ satisfies Condition $(\mathrm{K})$, all the ideals of the Leavitt path algebra $L_{K}(E)$ are graded; in particular, they are generated by hereditary and saturated subsets of vertices of $E^{0}$ ([23, Theorem 4.5]). Hence, $L_{K}(E), 0$ and I, the ideal generated by $v$, which is just the Socle of $L_{K}(E)$ ([?, Theorem 4.2]) are the only two-sided ideals of $L_{K}(E)$. Obviously, $L_{K}(E) / L_{K}(E)$ is flat as a left $L_{K}(E)$-module; by Corollary $2.10 L_{K}(E) / 0$ is flat and by Corollary $2.24 L_{K}(E) / I$ is also flat as a left $L_{K}(E)$-module. Now, right weakly regularity of the Leavitt path algebra $L_{K}(E)$ follows from Theorem 3.1.

However, not every Leavitt path algebra is right weakly regular.

Examples 3.3. Let $K$ be a field. The Leavitt path $K$-algebras of the following graphs are not right weakly regular:

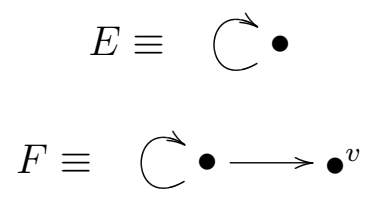

Indeed, in the first case, the associated Leavitt path algebra is isomorphic to $K\left[x, x^{-1}\right]$, which is not right weakly regular as not every (right) ideal is idempotent: take, for example, the ideal $I$ generated by $1+x$; it is not idempotent as otherwise $1+x \in I^{2}$ would imply $1+x=f(1+x)^{2}$ for some $f \in K\left[x, x^{-1}\right]$, equivalently, $1=f(1+x)$, which is not possible as $1+x$ is not invertible in $K\left[x, x^{-1}\right]$.

In the second case, denote by $T$ the corresponding Leavitt path algebra (which is the Toeplitz algebra) and by $I$ the ideal generated by the vertex $v$. If $T$ were right weakly regular, then $T / I$ would be right weakly regular (use [40, Proposition 5$]$ ), but $T / I$ is isomorphic to $L_{K}(E)$ (apply [23, Lemma $\left.2.3(1)\right]$ ), which is not right weakly regular. 
The following theorem follows from results by K. R. Goodearl [32] and M. Tomforde [43] and will be of importance to characterize right weakly regular Leavitt path algebras.

Theorem 3.4. Let $K$ be a field and $E$ an arbitrary graph. Then the following are equivalent conditions:

(i) Every ideal of $L_{K}(E)$ is graded.

(ii) The Leavitt path algebra $L_{K}(E)$ is exchange.

(iii) The graph E satisfies Condition (K).

Proof. (ii) $\Leftrightarrow$ (iii) is [32, Theorem 4.2], while (i) $\Leftrightarrow$ (iii) is [43, Theorem 6.16].

Other crucial step in the characterization of right weakly regular Leavitt path algebras will be Proposition 3.7, which states that every graded ideal in a Leavitt path algebra is itself (isomorphic to) a Leavitt path algebra. This is the algebraic analog of [28, Lemma 1.6] and a generalization of [18, Lemma 1.2] to arbitrary graphs. To prove it, we need first some definitions and notation. We will follow [43] and [28].

Definitions 3.5. Let $E$ be an arbitrary graph and $H$ a hereditary subset of $E^{0}$; we say that a vertex is a breaking vertex of $H$ if it belongs to the set

$$
B_{H}:=\left\{v \in E^{0} \backslash H \quad \mid v \in E_{\text {inf }}^{0} \quad \text { and } \quad 0<\left|s^{-1}(v) \cap r^{-1}\left(E^{0} \backslash H\right)\right|<\infty\right\} .
$$

For $v \in B_{H}$, define and denote by $I_{(H, S)}$ the ideal of $L_{K}(E)$ generated by $H \cup\left\{v^{H} \mid v \in S\right\}$.

According to [43, Theorem 5.7], all the graded ideals of a Leavitt path algebra $L_{K}(E)$ are of the form $I_{(H, S)}$ for a suitable pair $(H, S)$, being $H$ a hereditary and saturated subset of vertices of $E^{0}$.

Let $E$ be a graph, and let $\emptyset \neq H$ be a hereditary and saturated subset of $E^{0}$ and let $S \subseteq B_{H}$. Denote by $\widetilde{F}_{E}(H, S)$ the collection of all finite paths $\alpha=e_{1}, \ldots, e_{n}$ of positive length such that $e_{i} \in E^{1}, s(\alpha) \in E^{0} \backslash H, r(\alpha) \in H \cup S$ and $r\left(\alpha_{i}\right) \in E^{0} \backslash(H \cup S)$ for $i<n$.

We define

$$
F_{E}(H, S)=\widetilde{F}_{E}(H, S) \backslash\left\{e \in E^{1} \mid s(e) \in S \text { and } r(e) \in H\right\} .
$$

We denote by $\bar{F}_{E}(H, S)$ another copy of $F_{E}(H, S)$. For $\alpha \in F_{E}(H, S)$, we write $\bar{\alpha}$ to denote a copy of $\alpha$ in $\bar{F}_{E}(H, S)$. Then, we define the graph ${ }_{H} E_{S}=\left({ }_{H} E_{S}^{0},{ }_{H} E_{S}^{1}, s^{\prime}, r^{\prime}\right)$ as follows:

$$
\begin{aligned}
& { }_{H} E_{S}^{0}=\left({ }_{H} E_{S}\right)^{0}:=H \cup S \cup F_{E}(H, S) . \\
& { }_{H} E_{S}^{1}=\left({ }_{H} E_{S}\right)^{1}:=\left\{e \in E^{1} \mid s(e) \in H\right\} \cup\left\{e \in E^{1} \mid s(e) \in S \text { and } r(e) \in H\right\} \cup \bar{F}_{E}(H, S) .
\end{aligned}
$$

For every $\alpha \in F_{E}(H, S), s^{\prime}(\bar{\alpha})=\alpha$ and $r^{\prime}(\bar{\alpha})=r(\alpha)$.

For the other edges of ${ }_{H} E_{S}^{1}, s^{\prime}(e)=s(e)$ and $r^{\prime}(e)=r(e)$.

Remark 3.6. The graph ${ }_{H} E_{S}$ we have just described has the following properties:

(i) It contains the restriction graph $E_{H}=\left(H,\left\{e \in E^{1} \mid s(e) \in H\right\},\left.r\right|_{\left(E_{H}\right)^{1}},\left.s\right|_{\left(E_{H}\right)^{1}}\right)$.

(ii) Every vertex in $S \subseteq{ }_{H} E_{S}^{0}$ emits infinitely many edges into $H$ and does not emit other edges. 
(iii) Every vertex in $F_{E}(H, S)$ emits exactly one edge, which ends in $H \cup S$, thus every cycle in the new graph ${ }_{H} E_{S}$ comes from a cycle in the restriction graph $E_{H}$ and therefore the cycle structure of ${ }_{H} E_{S}$ is the same as that of $E_{H}$. Concretely:

- If $E_{H}$ satisfies Condition (L), then so does ${ }_{H} E_{S}$.

- If $E_{H}$ satisfies Condition $(\mathrm{K})$, then so does ${ }_{H} E_{S}$.

We are now ready to show that every graded ideal in a Leavitt path algebra over an arbitrary graph can also be realized as a Leavitt path algebra for some graph.

Proposition 3.7. Let $E$ be an arbitrary graph and $K$ any field. Then, for any graded ideal $I=I_{(H, S)}$ of the Leavitt path algebra $L_{K}(E)$, there exists a graded isomorphism $\varphi$ : $L_{K}\left({ }_{H} E_{S}\right) \rightarrow I_{(H, S)}$.

Proof. Define $\varphi$ as the map that acts as follows:

$$
\begin{aligned}
& \varphi(u)=u \text { for every } u \in H, \\
& \varphi(v)=v^{H} \text { for every } v \in S, \\
& \varphi(\alpha)= \begin{cases}\alpha \alpha^{*} & \text { if } r(\alpha) \in H\left(\alpha \in F_{E}(H, S)\right) \\
\alpha r(\alpha)^{H} \alpha^{*} & \text { if } r(\alpha) \in S\left(\alpha \in F_{E}(H, S)\right),\end{cases} \\
& \varphi(e)=e \text { for every } e \in E^{1} \text { with } s(e) \in H, \\
& \varphi(f)=f \text { for every } f \in E^{1} \text { with } r(f) \in H \text { and } s(f) \in S, \\
& \varphi(\bar{\alpha})= \begin{cases}\alpha & \text { if } r(\alpha) \in H\left(\alpha \in F_{E}(H, S)\right) \\
\alpha r(\alpha)^{H} & \text { if } r(\alpha) \in S\left(\alpha \in F_{E}(H, S)\right),\end{cases}
\end{aligned}
$$

It is not difficult to show that there exists an $*$-homomorphism of $K$-algebras (in fact, by the definition, it is a graded homomorphism) that extends $\varphi$.

To show the injectivity we will apply [22, Theorem 3.7], which remains valid for any graph and any field. Hence, we have to prove that $\varphi$ sends every vertex of ${ }_{H} E_{S}^{0}$ to a non-zero element of the ideal $I_{(H, S)}$ and every cycle without exits $c$ in ${ }_{H} E_{S}$ to a non-nilpotent homogeneous element of non-zero degree in $I_{(H, S)}$.

Indeed, the condition on the vertices of ${ }_{H} E_{S}^{0}$ is fulfilled, and to show the condition on the cycles without exits, take into account Remark 3.6 (iii) and the definition of $\varphi$ (both say that the image of a cycle without exits in ${ }_{H} E_{S}$ is the cycle itself but seen inside the ideal $I_{(H, S)}$ ).

Now we show that $\varphi$ is surjective. It was proved in [43, Lemma 5.6] that $I_{(H, S)}$ is generated (as a $K$-vector space) by elements $\alpha \beta^{*}$, with $r(\alpha)=r(\beta) \in H$ and $\alpha v^{H} \beta^{*}$, with $r(\alpha)=$ $r(\beta)=v \in S$ (being $\alpha, \beta$ paths in $L_{K}(E)$ ), therefore to check the surjectivity it is enough to find inverse images of the elements of the form $\alpha$ or $\beta r(\beta)^{H}$, for $\alpha, \beta$ paths in $E$ with $r(\alpha) \in H$ and $r(\beta) \in S$.

To start with, choose a path $\alpha$ in $E$ such that $r(\alpha) \in H$, say $\alpha=f_{1} \ldots f_{m}$, with $f_{i} \in E^{1}$. If $r(\alpha) \in H$, then $\alpha$ is a path in the restriction graph and by the definition of $\varphi$ we have $\alpha=\varphi(\alpha) \in \operatorname{Im}(\varphi)$. Suppose $(r \alpha) \notin H$. 
Now $r\left(f_{m}\right) \in H$. If $r\left(f_{1}\right) \in H$ then $\varphi\left(f_{i}\right)=f_{i}$ for all $i=2, \ldots, n$ and $f_{1}=\varphi\left(f_{1}\right)$ or $\varphi\left(\overline{f_{1}}\right)$ according as $s\left(f_{1}\right) \in S$ or $s\left(f_{1}\right) \notin S$ (hence $f_{1} \in F_{E}(H, S)$ ). In either case, it is clear that $\alpha \in \operatorname{Im}(\varphi)$. Suppose $r\left(f_{1}\right) \notin H$.

Let $n$ be the smallest integer such that $1<n \leq m$ and $r\left(f_{n}\right) \in H$ (and so $r\left(f_{n-1}\right) \notin H$ ). We distinguish two cases. Suppose $s\left(f_{n}\right) \in S$. Since any edge from a vertex in $S$ must end in a vertex in $H$ (see Remark 3.6 (ii)), $s\left(f_{i}\right) \notin S$ (and $\notin H$ ) for $i=1, \ldots, n-1$. Thus $\gamma=f_{1} \cdots f_{n-1} \in F_{E}(H, S)$ and we get $\alpha=\gamma \cdot f_{n} \cdots f_{m}=\varphi(\gamma) \varphi\left(f_{n}\right) \ldots \varphi\left(f_{m}\right) \in \operatorname{Im}(\varphi)$. On the other hand if $s\left(f_{n}\right) \notin S$ and hence $\notin H \cup S$, then again by Remark 3.6 (ii), $r\left(f_{i}\right) \notin$ $H \cup S$ for $i=1, \ldots, n-1$. Thus $\delta=f_{1} \cdots f_{n} \in F_{E}(H, S)$ and $\alpha=\delta \cdot f_{n+1} \cdots f_{m}=$ $\varphi(\delta) \varphi\left(f_{n+1}\right) \ldots \varphi\left(f_{m}\right) \in \operatorname{Im}(\varphi)$.

To finish the proof, consider a path $\beta=e_{1} \ldots e_{n}$ of length $n$ with $r(\beta) \in S$. Since $H$ is hereditary and $H \cap S=\emptyset, s\left(e_{i}\right) \notin H$ for $i=1, \ldots, n$. Since $r\left(e_{n}\right) \in S$, we also conclude, by Remark 3.6 (ii), that $s\left(e_{i}\right) \notin S$ for all $i=1, \ldots, n$. This means that $\beta \in F_{E}(H, S)$ and so, by definition, $\beta r(\beta)^{H}=\varphi(\bar{\beta})$. This proves that $\varphi$ is surjective.

Proposition 3.8. Let $E$ be an arbitrary graph and $K$ be any field. If $E$ satisfies Condition $(\mathrm{K})$, then the Leavitt path algebra $L_{K}(E)$ is right weakly regular.

Proof. Let $I$ be an ideal of $L_{K}(E)$. By Theorem 3.4 the ideal $I$ is graded and by Proposition 3.7 it is isomorphic, as a $K$-algebra, to a Leavitt path algebra. Since every Leavitt path algebra has local units, $I$ itself has local units.

Now we prove that $I$ is pure, i.e., that it satisfies Condition (ii) in Theorem 2.25; observe that it can be applied by virtue of Example 2.12. To this end, consider an element $a \in I$; use that $I$ has local units to find an idempotent $u \in I$ such that $a=a u$. Then

$$
\begin{array}{cl}
\rho_{u}: L_{K}(E) & \rightarrow I \\
r & \mapsto r u
\end{array}
$$

is a homomorphism of left $L_{K}(E)$-modules such that $\rho_{u}(a)=a u=a$, hence $L_{K}(E) / I$ is flat as a left $L_{K}(E)$-module and by Theorem $3.1, L_{K}(E)$ is right weakly regular.

Proposition 3.9. Let $E$ be a row-finite graph and $K$ be an arbitrary field. If the Leavitt path algebra $L_{K}(E)$ is right weakly regular, then the graph $E$ satisfies Condition $(\mathrm{K})$.

Proof. We show first that if the Leavitt path algebra associated to a graph $E$ is right weakly regular, then the graph satisfies Condition (L).

Suppose that $c$ is a cycle without exits in $E$, and let $H$ be the saturated closure of $c^{0}$. Follow [6, Proposition 3.5 (iii)] to obtain that $I(H)$ is isomorphic to $M_{n}\left(K\left[x, x^{-1}\right]\right)$ for some $n \in \mathbb{N} \cup\{\infty\}$. By [40, Proposition 5], since $L_{K}(E)$ is right weakly regular $I(H)$ is right weakly regular, that is, $M_{n}\left(K\left[x, x^{-1}\right]\right)$ is right weakly regular. On the other hand, by Proposition 3.11 (i) every corner of $M_{n}\left(K\left[x, x^{-1}\right]\right)$ is right weakly regular. But for $e$ the matrix unit in $M_{n}\left(K\left[x, x^{-1}\right]\right)$ having all the entries equal to zero except in the entry $(1,1)$, the corner $e M_{n}\left(K\left[x, x^{-1}\right]\right) e$ is isomorphic to $K\left[x, x^{-1}\right]$, which is not right weakly regular, as we explained before. This contradiction shows that $E$ has no cycles without exits.

Now we prove that right weakly regularity for a Leavitt path algebra implies Condition $(\mathrm{K})$.

Suppose on the contrary that there exists a vertex $v$ and a cycle $c=e_{1} \ldots e_{n}$ based at $v$, with $\operatorname{card}(C S P(v))=1$ and consider the sets $A=\left\{e \in E^{1} \mid e\right.$ exit of $\left.c\right\}, B=\{r(e) \mid e \in A\}$, 
and let $H$ be the hereditary saturated closure of $B$. With a similar argument to that used in [4, pp. 557 and 558 (proof of Lemma 7)] we obtain that $H \cap \alpha^{0}=\emptyset$; this means that $H$ is a proper subset of $E^{0}$. Moreover, $c^{0} \subseteq(E / H)^{0}$ and $\left\{e_{1}, \ldots, e_{n}\right\} \subseteq(E / H)^{1}$, whence $c$ is a cycle in $E / H$ with no exits.

Since $L_{K}(E / H) \cong L_{K}(E) / I(H)([23$, Lemma $2.3(1)]), L_{K}(E / H)$ is a right weakly regular ring [40, Proposition 5] and, by the previous step, $E / H$ satisfies Condition (L), a contradiction.

The authors are thankful to Gene Abrams for the following remark.

Remark 3.10. Proposition 3.9 can be generalized in the following way: if $\mathcal{P}$ is a ringtheoretic property of $L_{K}(E)$ that implies Condition (L) on the graph $E$, and is closed under homomorphic images, then $\mathcal{P}$ implies Condition $(\mathrm{K})$ on the graph $E$ too.

The property of being right weakly regular is preserved by corners and by matrices. This fact is the first step to show that right weakly regularity is a Morita invariant property for rings with local units.

Proposition 3.11. Let $R$ be a ring.

(i) If $R$ is right weakly regular then, for every idempotent $e \in R$, the corner eRe is a right weakly regular ring.

(ii) If $R$ has local units then $R$ is right weakly regular if and only if for every idempotent $e \in R$, the corner eRe is a right weakly regular ring.

(iii) $R$ is right weakly regular if and only if for every natural $n$ the matrix ring $M_{n}(R)$ is a right weakly regular ring.

Proof. To show that if $R$ is a right weakly regular ring then every corner and every matrix ring over $R$ is right weakly regular, follow the proof of the analogue results for unital rings in [44, Proposition 20.4] and apply Theorem 3.1.

It is a tautology that if every matrix ring over $R$ is a right weakly regular ring then $R$ is right weakly regular (take $n=1$ ).

Finally, to finish the proof of (ii) suppose that every corner in $R$ is right weakly regular, and consider an element $a \in R$. Apply that $R$ has local units to find an idempotent $e \in R$ such that $a \in e R e$. By the hypothesis, $e R e$ is a right weakly regular ring so (Theorem 3.1) there exists $x \in e$ ReaeRe $\subseteq R a R$ such that $a=a x$. By Theorem 3.1 this means that $R$ is a right weakly regular ring.

We next recall the notion of Morita equivalence for idempotent rings (a ring $R$ is said to be idempotent if $R^{2}=R$ ).

Let $R$ and $S$ be two rings, ${ }_{R} N_{S}$ and ${ }_{S} M_{R}$ two bimodules and $(-,-): N \times M \rightarrow R$, $[-,-]: M \times N \rightarrow S$ two maps. Then the following conditions are equivalent:

(i) $\left(\begin{array}{cc}R & N \\ M & S\end{array}\right)$ is a ring with componentwise sum and product given by:

$$
\left(\begin{array}{cc}
r_{1} & n_{1} \\
m_{1} & s_{1}
\end{array}\right)\left(\begin{array}{cc}
r_{2} & n_{2} \\
m_{2} & s_{2}
\end{array}\right)=\left(\begin{array}{cc}
r_{1} r_{2}+\left(n_{1}, m_{2}\right) & r_{1} n_{2}+n_{1} s_{2} \\
m_{1} r_{2}+s_{1} m_{2} & {\left[m_{1}, n_{2}\right]+s_{1} s_{2}}
\end{array}\right)
$$


(ii) $[-,-]$ is $S$-bilinear and $R$-balanced, $(-,-)$ is $R$-bilinear and $S$-balanced and the following associativity conditions hold:

$$
(n, m) n^{\prime}=n\left[m, n^{\prime}\right] \quad \text { and } \quad[m, n] m^{\prime}=m\left(n, m^{\prime}\right),
$$

for all $m, m^{\prime} \in M$ and $n,{ }^{\prime} \in N$.

That $[-,-]$ is $S$-bilinear and $R$-balanced and that $(-,-)$ is $R$-bilinear and $S$-balanced is equivalent to having bimodule maps $\varphi: N \otimes_{S} M \rightarrow R$ and $\psi: M \otimes_{R} N \rightarrow S$, given by

$$
\varphi(n \otimes m)=(n, m) \quad \text { and } \quad \psi(m \otimes n)=[m, n]
$$

so that the associativity conditions above read

$$
\varphi(n \otimes m) n^{\prime}=n \psi\left(m \otimes n^{\prime}\right) \quad \text { and } \quad \psi(m \otimes n) m^{\prime}=m \varphi\left(n \otimes m^{\prime}\right) .
$$

A Morita context is a sextuple $(R, S, N, M, \varphi, \psi)$ satisfying one of the (equivalent) conditions given above. The associated ring (in condition (i)) is called the Morita ring of the context. By abuse of notation we will write $(R, S, N, M)$ instead of $(R, S, N, M, \varphi, \psi)$ and will identify $R, S, N$ and $M$ with their natural images in the Morita ring associated to the context. The Morita context is said to be surjective if the maps $\varphi$ and $\psi$ are both surjective.

In classical Morita theory, it is shown that two rings with identity $R$ and $S$ are Morita equivalent (i.e., $R$-Mod and $S$-Mod are equivalent categories) if and only if there exists a surjective Morita context $(R, S, N, M, \varphi, \psi)$. The approach to Morita theory for rings without identity by means of Morita contexts appears in a number of papers (see [31] and the references therein) in which many consequences are obtained from the existence of a surjective Morita context for two rings $R$ and $S$.

For an idempotent ring $R$ we denote by $R$-Mod the full subcategory of the category of all left $R$-modules whose objects are the "unital" nondegenerate modules. Here, a left $R$-module $M$ is said to be unital if $M=R M$, and $M$ is said to be nondegenerate if, for $m \in M$, $R m=0$ implies $m=0$. Note that, if $R$ has an identity, then $R$-Mod is the usual category of left $R$-modules.

It is shown in [36, Theorem] that, if $R$ and $S$ are arbitrary rings having a surjective Morita context, then the categories $R$-Mod and $S$-Mod are equivalent. The converse direction is proved in [31, Proposition 2.3] for idempotent rings, yielding the following theorem.

Theorem 3.12. Let $R$ and $S$ be two idempotent rings. Then the categories $R$-Mod and $S$-Mod are equivalent if and only if there exists a surjective Morita context $(R, S, N, M)$.

Given two idempotent rings $R$ and $S$, we will say that they are Morita equivalent if the categories $R$-Mod and $S$-Mod are equivalent.

Theorem 3.13. Let $R$ and $S$ be rings with local units that are Morita equivalent. Then $R$ is right weakly regular if and only if $S$ is right weakly regular.

Proof. Let $(R, S, N, M)$ be a surjective Morita context and assume that $R$ is a right weakly regular ring. We are going to show that for every idempotent $e$ in $S$ the corner $e S e$ is a right weakly regular ring. The result then will follow by Proposition 3.11 (ii).

Since $e \in S=M N$, we can find $x_{1}, \ldots, x_{n} \in M, y_{1}, \ldots, y_{n} \in N$ satisfying $e=\sum_{i=1}^{n} x_{i} y_{i}$. Put $\mathbf{x}=\left(x_{1}, \ldots, x_{n}\right), \mathbf{y}=\left(y_{1}, \ldots, y_{m}\right)$. Then $e=\mathbf{x y}^{t}$, and we may assume $x_{i}=e x_{i}$ and $y_{i}=y_{i} e$, for every $i \in\{1, \ldots, n\}$. Note that the element $\mathbf{y}^{t} \mathbf{x} \mathbf{y}^{t} \mathbf{x}$ is an idempotent in $M_{n}(R)$ : $\mathbf{y}^{t} \mathbf{x} \mathbf{y}^{t} \mathbf{x} \mathbf{y}^{t} \mathbf{x} \mathbf{y}^{t} \mathbf{x}=\mathbf{y}^{t}\left(\mathbf{x} \mathbf{y}^{t}\right)\left(\mathbf{x} \mathbf{y}^{t}\right)\left(\mathbf{x y}^{t}\right) \mathbf{x}=\mathbf{y}^{t} e^{3} \mathbf{x}=\mathbf{y}^{t} e \mathbf{x}=\mathbf{y}^{t} \mathbf{x} \mathbf{y}^{t} \mathbf{x}$. 
Consider the map

$$
\varphi: \quad \begin{array}{clc}
M_{n}(R)_{\mathbf{y}^{t} \mathbf{x} \mathbf{y}^{t} \mathbf{x}} & \rightarrow & e S e \\
\left(\mathbf{y}^{t} \mathbf{x} \mathbf{y}^{t} \mathbf{x}\right) a\left(\mathbf{y}^{t} \mathbf{x} \mathbf{y}^{t} \mathbf{x}\right) & \mapsto \mathbf{x y}^{t} \mathbf{x} a \mathbf{y}^{t} \mathbf{x} \mathbf{y}^{t},
\end{array}
$$

which is easily seen to be a ring isomorphism. Since matrix rings over right weakly regular rings are again right weakly regular (Proposition 3.11 (iii)) we obtain (via Proposition 3.11 (i)) that $e S e$ is a right weakly regular ring.

Proposition 3.14. Let $E$ be a countable but not necessarily row-finite graph, and let $K$ be any field. Then $E$ satisfies Condition $(\mathrm{K})$ if and only if the Leavitt path algebra $L_{K}(E)$ is right weakly regular.

Proof. If $E$ satisfies Condition $(\mathrm{K})$ then $L_{K}(E)$ is right weakly regular by Proposition 3.8.

For the converse, suppose that $L_{K}(E)$ is a right weakly regular ring. Given the countable graph $E$, denote by $F$ the desingularization of $E$ (see the construction, for example, in [5]). By [5, Theorem 5.2], the Leavitt path algebras $L_{K}(E)$ and $L_{K}(F)$ are Morita equivalent idempotent rings. Since right weakly regularity is a Morita invariant property for rings with local units (Theorem 3.13), the Leavitt path algebra $L_{K}(F)$ is right weakly regular, therefore (any desingularization is a row-finite graph so Proposition 3.9 can be applied) $F$ satisfies Condition $(\mathrm{K})$ and (equivalently) $L_{K}(F)$ is an exchange ring (Theorem 3.4). Use now that the exchange property is Morita invariant for rings with local units [16, Theorem 2.1] to obtain that the Leavitt path algebra $L_{K}(E)$ is an exchange ring, hence (Theorem 3.4) the graph $E$ satisfies Condition (K).

Theorem 3.15. Let $K$ be any field and $E$ an arbitrary graph. Then, the following are equivalent conditions:

(i) The Leavitt path algebra $L_{K}(E)$ is a right weakly regular ring.

(ii) The graph $E$ satisfies Condition $(\mathrm{K})$.

(iii) The Leavitt path algebra $L_{K}(E)$ is a left weakly regular ring.

(iv) The Leavitt path algebra $L_{K}(E)$ is an exchange ring.

(v) Every ideal of $L_{K}(E)$ is graded.

(vi) Every ideal of $L_{K}(E)$ is isomorphic to a Leavitt path algebra.

(vii) Every ideal of $L_{K}(E)$ has local units.

Proof. The equivalences (ii) $\Leftrightarrow$ (iv) $\Leftrightarrow$ (v) have been stated in Theorem 3.4.

(i) $\Leftrightarrow$ (iii) follows because if $I$ is any left/right ideal then $I^{*}$ is a right/left ideal and $I^{*}$ is idempotent if and only if $I$ is idempotent.

(i) $\Rightarrow$ (ii). For countable graphs it has been settled in Proposition 3.9. Now we will prove the result for arbitrary graphs following the same sketch as Goodearl in the proof of $(\Rightarrow)$ in [32, Theorem 4.2].

Write $(K, E)=\underline{\lim }\left(K_{\alpha}, E_{\alpha}\right)$ and $L_{K}(E)=\underline{\lim } L_{K_{\alpha}}\left(E_{\alpha}\right)$ as in [32, (3.5)], with $E_{\alpha}$ a countable graph for every $\alpha \in A$. Given $\alpha \in A$ and $a \in L_{K_{\alpha}}\left(E_{\alpha}\right)$, since $L_{K}(E)$ is a right weakly regular ring, by Theorem 3.1 there exist a finite number of elements $x, x_{i}, y_{i} \in L_{K}(E)$ such that $L\left(\eta_{\alpha}\right)(x)=\sum x_{i} a y_{i}$ and $a=a L\left(\eta_{\alpha}\right)(x)$ (here we are following the same notation as in [32]). And there exist $\gamma \geq \alpha$ in $A$ and $x_{i}^{\prime}, y_{i}^{\prime} \in L_{K_{\gamma}}\left(E_{\gamma}\right)$ such that $L\left(\phi_{\alpha \gamma}\right)(x)=\sum x_{i}^{\prime} a y_{i}^{\prime}$ and $a=a L\left(\phi_{\alpha \gamma}\right)(x)$. By the (so called in [32]) Modus Operandi, it follows that for each $\alpha \in A$ 
there exists some $\beta \geq \alpha$ in $A$ such that $L_{K_{\beta}}\left(E_{\beta}\right)$ is a right weakly regular ring. For each such $\beta$ the graph $E_{\beta}$ satisfies Condition (K) by Proposition 3.9, hence $E$ satisfies Condition (K).

(ii) $\Rightarrow$ (vi). If $E$ satisfies Condition (K), by [32, Theorem 3.8] any ideal $I$ is graded, hence, by Proposition 3.7, $I$ is isomorphic to a Leavitt path algebra.

(vi) $\Rightarrow$ (vii) because every Leavitt path algebra has local units.

(vii) $\Rightarrow$ (i). Suppose that every ideal of the Leavitt path algebra $L_{K}(E)$ has local units. Consider $a \in L_{K}(E)$. By the hypothesis the ideal $L_{K}(E) a L_{K}(E)$ has local units, so there exists $u^{2}=u \in L_{K}(E) a L_{K}(E)$ such that $a=a u$. By Theorem 3.1 the Leavitt path algebra is right weakly regular.

Although Theorem 3.15 shows that the notions of exchange ring and right weakly regular ring are equivalent for Leavitt path algebras, this is not true for a general ring. We finish this section by displaying examples of such situations.

Examples 3.16. (i) A right weakly regular ring need not be an exchange ring. Consider $R=W_{1}[K]$, the first Weyl algebra over a field $K$ of characteristic zero ( $R$ can be seen as $K[x, y]$, where $x$ and $y$ satisfy the conditions $x y-y x=1)$. Then $R$ is a simple domain (not a division ring) which is not an exchange ring (see [33, Remark to Proposition 1.8]).

(ii) An exchange ring is not necessarily weakly regular. Indeed, let $N$ be a nilpotent ring and $K$ a field. Then $R=N \oplus M_{2}(K)$ is an exchange ring (because both $N$ and $M_{2}(K)$ are exchange rings) while it is not right weakly regular, as not every right ideal of $R$ is idempotent.

\section{Self-injective Leavitt Path algebras}

In this section we will show that a Leavitt path algebra $L_{K}(E)$ is left (right) self-injective if and only if $E$ is a row-finite acyclic graph in which every infinite path contains a line point and so, if and only if $L_{K}(E)$ is a semisimple ring, that is, a direct sum of simple one-sided ideals.

Our first result shows that a self-injective $L_{K}(E)$ must be von Neumann regular.

Proposition 4.1. Let $E$ be an arbitrary graph and $K$ any field. If $L_{K}(E)$ is left (right) self-injective then $L_{K}(E)$ is von Neumann regular and $E$ acyclic.

Proof. If $\epsilon$ is any idempotent in $L_{K}(E)$, then $L_{K}(E) \epsilon$, being a summand, is an injective module. Now $\epsilon L_{K}(E) \epsilon \cong E_{n d} d_{L_{K}(E)}\left(L_{K}(E) \epsilon\right)$ satisfies $\epsilon L_{K}(E) \epsilon / J\left(\epsilon L_{K}(E) \epsilon\right)$ is von Neumann regular, where $J\left(\epsilon L_{K}(E) \epsilon\right)$ is the Jacobson radical of $\epsilon L_{K}(E) \epsilon$ (apply that $\epsilon R \epsilon$ is left (right) self-injective and [37, Corollary $13.2(2)]$ ). Now, by [34, Proposition 1, Page 48] (which is true for non-unital rings), $J\left(\epsilon L_{K}(E) \epsilon\right)=\epsilon L_{K}(E) \epsilon \cap J\left(L_{K}(E)\right)=\epsilon J\left(L_{K}(E)\right) \epsilon$. By [5, Proposition 6.3] and [8, Proposition 1.8], $J\left(L_{K}(E)\right)=0$ and therefore $J\left(\epsilon L_{K}(E) \epsilon\right)=0$. We thus conclude that for any idempotent $\epsilon$ in $L_{K}(E), \epsilon L_{K}(E) \epsilon$ is a von Neumann regular ring. Since $L_{K}(E)$ is a ring with local units, for every $a \in L_{K}(E)$, there is an idempotent $\epsilon$ such that $\epsilon a=a=a \epsilon$. Since $a \in \epsilon L_{K}(E) \epsilon$, there is a $b \in \epsilon L_{K}(E) \epsilon$ such that $a b a=a$. Hence $L_{K}(E)$ is von Neumann regular. By [7, Theorem 1] this is equivalent to the graph $E$ being acyclic.

We shall show that the left self-injectivity of $L_{K}(E)$ implies that $E$ is a row-finite graph. Even though a great amount of algebraic properties of $L_{K}(E)$ have been characterized in terms of properties of the graph (and, depending on the type of graphs considered, an overview of 
these works are: $[3,4,6,17,18, ?, 23]$ for the row-finite case, $[5,22,42]$ for the countable but not necessarily row-finite setting, and $[8,43,32]$ for arbitrary graphs), this is the first instance where, starting from an arbitrary graph $E$, an algebraic property of $L_{K}(E)$ automatically implies row-finiteness of the graph $E$.

We begin with some preparatory lemmas. Lemma 4.3 states a useful, perhaps known, fact. We give its proof for the sake of completeness.

Following T. Jech [35], a family $\mathcal{F}$ of subsets in a set $X$ with cardinality $\sigma$ is called an independent family if given any finitely many distinct subsets $X_{1}, \ldots, X_{m}, Y_{1}, \ldots, Y_{n}$ in $\mathcal{F}$, the cardinality of $X_{1} \cap \cdots \cap X_{m} \cap\left(X \backslash Y_{1}\right) \cap \cdots \cap\left(X \backslash Y_{n}\right)$ is $\sigma$.

Lemma 4.2. [35, Lemma 7.7]. A set $X$ of infinite cardinal $\sigma$ contains a family of $2^{\sigma}$ independent subsets.

Lemma 4.3. Let $\sigma$ be an infinite cardinal and let $K$ be any field. Then the $K$-dimension of the direct product $P$ of $\sigma$ copies of $K$ is $2^{\sigma}$.

Proof. We view $P$ as the set of all functions from a set $X$ of cardinality $\sigma$ to $K$. By Lemma 4.2 , there is an independent family $\mathcal{F}$ of $2^{\sigma}$ subsets of $X$. It is then clear that the set $\left\{\chi_{A} \mid A \in \mathcal{F}\right\}$, where $\chi_{A}$ is the characteristic function on $A$, is a $K$-independent subset of $P$ and hence $P$ has $K$-dimension $2^{\sigma}$.

Proposition 4.4. If a Leavitt path algebra $L_{K}(E)$ is left (right) self-injective then the graph E must be row-finite.

Proof. Suppose, by way of contradiction, the graph $E$ contains a vertex $v$ which emits infinitely many edges. For each positive integer $n$, let $\alpha_{n}$ be the cardinality of the set $Y_{n}$ of all paths of length $n$ that begin with $v$. Then the set $Y$ of all finite paths that begin with $v$ has infinite cardinality $\sigma=\sup \left\{\alpha_{n} \mid n=1,2,3, \ldots\right\}$. Now every element of $v L_{K}(E) v$ is a finite sum of the form $\sum_{i=1}^{n} k_{i} p_{i} q_{i}^{*}$ where $p_{i}$ and $q_{i}$ are finite paths beginning with the vertex $v$ and $k_{i} \in K$. Since the cardinality of the set of all finite subsets $Y$ is again $\sigma$, we conclude that the $K$-dimension of $v L_{K}(E) v$ is $\leq \sigma$.

Next we wish to choose a subset $X$ of $Y$ of cardinality $\sigma$ such that the following left ideals $\left\{L_{K}(E) p p^{*} \mid p \in X\right\}$ are $L_{K}(E)$-independent, that is, for all $p \in X, L_{K}(E) p p^{*} \cap$ $\sum_{q \in X, q \neq p} L_{K}(E) q q^{*}=0$.

First observe that, for each $n$, the set $\left\{L_{K}(E) p p^{*} \mid p \in Y_{n}\right\}$ is $L_{K}(E)$-independent: if $p_{1}, \ldots, p_{k} \in Y_{n}$ and $r_{i} p_{i} p_{i}^{*}=\sum_{j \neq i} r_{j} p_{j} p_{j}^{*}$ then $r_{i} p_{i} p_{i}^{*} p_{i}=\sum_{j \neq i} r_{j} p_{j} p_{j}^{*} p_{i}=0$, since $p_{i} \neq p_{j}$ and $p_{i}, p_{j}$ all have the same length. Thus $r p_{i}=r_{i} p_{i} p_{i}^{*} p_{i}=0$ and hence $r_{i} p_{i} p_{i}^{*}=0$ proving the $L_{K}(E)$-independence.

So if $\alpha_{n}=\left|Y_{n}\right|=\sigma$ for some $n$, then we choose $X=Y_{n}$.

Suppose $\alpha_{n}<\sigma$ for all $n$. Observe that $\alpha_{n}$ need not always be $<\alpha_{n+1}$ since not every path in $Y_{n}$ need be a subpath of a path in $Y_{n+1}$. So we choose a subsequence $\left\{\alpha_{i_{n}} \mid n<\omega\right\}$ as follows. Let $\alpha_{i_{1}}=\alpha_{1}$. If $\alpha_{i_{n}}$ has been chosen for some $n \geq 1$, then choose $\alpha_{i_{n+1}}$ so that $i_{n+1}$ is the smallest integer such that $\alpha_{i_{n+1}}>\alpha_{i_{n}}$. Using the sequence $\left\{\alpha_{i_{n}}\right\}$, we begin constructing a sequence of sets $T_{n}$. Let $T_{1}=s^{-1}(v)=Y_{1}$. To construct the set $T_{2}$, we first note that, by the minimality of $i_{2}$, the number of paths of length less than $i_{2}$ is $<\alpha_{i_{2}}$ and that, for each $m<i_{2}$, the number of vertices in $r\left(Y_{m}\right)$ is $<\alpha_{i_{2}}$. Since $\left|Y_{\alpha_{2}-1}\right|<\alpha_{i_{2}}$, there must exist a 
vertex $v_{2} \in Y_{i_{2}-1}$ which emits $\alpha_{i_{2}}$ edges, say, $s^{-1}\left(v_{2}\right)=\left\{e_{\beta}^{(2)} \mid \beta<\alpha_{i_{2}}\right\}$. Let $\mu_{2}=f_{1}^{(2)} \ldots f_{i_{2}-1}^{(2)}$ be a path of length $i_{2}-1$ connecting $v$ to $v_{2}$. Then define

$$
T_{2}=\left\{\mu_{2} e_{\beta}^{(2)} \mid \beta<\alpha_{i_{2}}\right\} \cup\left(T_{1} \backslash\left\{\mu \mid \mu \text { is a subpath of } \mu_{2}\right\}\right) .
$$

In the definition of $T_{2}$, we are removing $\left\{\mu \mid \mu\right.$ is a subpath of $\left.\mu_{2}\right\}$, because we want to show (later) that the set $\left\{p p^{*} \mid p \in T_{2}\right\}$ is $L_{K}(E)$-independent. Suppose $T_{n}$ has been defined for all $n \geq 2$. By the arguments similar to those used in the construction of $T_{2}$, we conclude that there is a vertex $v_{n+1} \in r\left(Y_{i_{n}}\right)$ such that $v_{n+1}$ emits $\alpha_{i_{n+1}}$ edges. Let $s^{-1}\left(v_{n+1}\right)=\left\{e_{\beta}^{(n+1)} \mid \beta<\right.$ $\left.\alpha_{i_{n+1}}\right\}$ and let $\mu_{n+1}=f_{1}^{(n+1)} \ldots f_{i_{n+1}-1}^{(n+1)}$ be a path of length $i_{n+1}-1$ from $v$ to $v_{n+1}$. Define

$$
T_{n+1}=\left\{\mu_{n+1} e_{\beta}^{(n+1)} \mid \beta<\alpha_{i_{n+1}}\right\} \cup\left(T_{n} \backslash\left\{\mu \mid \mu \text { is a subpath of } \mu_{n+1}\right\}\right) .
$$

Thus $T_{n}$ is defined for all $n$.

We wish to show that, for a given $n$, the set $\left\{L_{K}(E) p p^{*} \mid p \in T_{n}\right\}$ is an independent set of left ideals of $L_{K}(E)$. First observe that, for all $p \neq q$ in $T_{n}$, we have that $p^{*} q=0$. This is because $p^{*} q \neq 0$ would imply $p=q \tau$ or $q=p \tau$ for some path $\tau$ and this is not possible by our construction. So if for some $p_{1}, \ldots, p_{k} \in T_{n}$ and $r_{1}, \ldots, r_{k} \in L_{K}(E)$, we have $r_{i} p_{i} p_{i}^{*}=$ $\sum_{j \neq i} r_{j} p_{j} p_{j}^{*}$, then multiplying on the right by $p_{i}$, we get $r_{i} p_{i}=r_{i} p_{i} p_{i}^{*} p_{i}=\sum_{j \neq i} r_{j} p_{j} p_{j}^{*} p_{i}=0$. Hence $r_{i} p_{i} p_{i}^{*}=0$, showing that $L_{K}(E) p_{i} p_{i}^{*} \cap \sum_{j \neq i} L_{K}(E) p_{j} p_{j}^{*}=0$.

But for $m \neq n$ and for $p_{i} \in T_{m}$ and $q_{j} \in T_{n}$, the set $\left\{L_{K}(E) p_{i} p_{i}^{*}, L_{K}(E) q_{j} q_{j}^{*}\right\}$ may not be $L_{K}(E)$-independent. So we modify the sets $T_{n}$ to obtain sets $W_{n}$ where the paths in different $W_{n}$ 's give rise to $L_{K}(E)$-independent left ideals. Accordingly, we define

$$
W_{1}=T_{1} \backslash\left\{f_{1}^{(m)} \mid m=2,3, \ldots\right\}
$$

and for any $n \geq 1$, define

$$
W_{n}=T_{n} \backslash\left\{f_{1}^{(m)} \ldots f_{i_{k}-1}^{(m)} \mid m=2,3, \ldots, \text { where } k=\min \{m, n\}\right\} .
$$

We now show that paths belonging to different $W_{n}$ 's are $L_{K}(E)$-independent. Suppose $p_{i} \in W_{i}$ for $i=1, \ldots, k$ such that $r_{i} p_{i} p_{i}^{*}=\sum_{j \neq i} r_{j} p_{j} p_{j}^{*}$. Then, as before $r_{i} p_{i}=r_{i} p_{i} p_{i}^{*} p_{i}=$ $\sum_{j \neq i} r_{j} p_{j} p_{j}^{*} p_{i}$. We claim $p_{j}^{*} p_{i}=0$. First note that $p_{j}^{*} p_{i} \neq 0$ implies that either $p_{i}=p_{j} \tau$ or $p_{j}=p_{i} \tau$ for some finite path $\tau$. Let $m$ and $n$ be the smallest integers such that $p_{i} \in T_{m}$ and $p_{j} \in T_{n}$ with $m<n$. By the minimality of the choice of $m$ and $n$, and the fact that $p_{j} \notin T_{m}$, $p_{j}$ must be of the form $\mu_{n} e_{\beta}^{(n)}$ for some $\beta<\alpha_{i_{n}}$. So if $p_{j}=p_{i} \tau$, then $p_{i}$ will be a subpath of $\mu_{n}$, which is not possible by construction. On the other hand $p_{i}=p_{j} \tau$ implies that $p_{j} \in W_{m}$, a contradiction to the minimal choice of $n$. Hence $p_{j}^{*} p_{i}=0$ and this shows, as before, that $L_{K}(E) p_{i} p_{i}^{*} \cap \sum_{j \neq i} L_{K}(E) p_{j} p_{j}^{*}=0$.

If we set $X=\cup_{n<\omega} W_{n}$, then $X$ has cardinality $\sigma$ and $\left\{L_{K}(E) p p^{*} \mid p \in X\right\}$ is an $L_{K}(E)$ independent family of left ideals contained in $L_{K}(E) v$.

Define $S=\sum_{p \in X} L_{K}(E) p p^{*}=\bigoplus_{p \in X} L_{K}(E) p p^{*} \subseteq L_{K}(E) v$. By hypothesis, the direct summand $L_{K}(E) v$ of $L_{K}(E)$ is injective and hence the exact sequence $0 \rightarrow S \stackrel{\phi}{\rightarrow} L_{K}(E) v$, where $\phi$ is the inclusion map, gives rise to an exact sequence

$$
\operatorname{Hom}_{L_{K}(E)}\left(L_{K}(E) v, L_{K}(E) v\right) \stackrel{\phi^{*}}{\rightarrow} \operatorname{Hom}_{L_{K}(E)}\left(S, L_{K}(E)\right) \rightarrow 0
$$


To see that this leads to a contradiction, first observe that

$$
\begin{aligned}
\operatorname{Hom}_{L_{K}(E)}\left(S, L_{K}(E)\right) & \supseteq \operatorname{Hom}_{L_{K}(E)}(S, S) \cong \operatorname{Hom}_{L_{K}(E)}\left(\bigoplus_{p \in X} L_{K}(E) p p^{*}, \bigoplus_{p \in X} L_{K}(E) p p^{*}\right) \\
& \cong \prod_{p \in X} \operatorname{Hom}_{L_{K}(E)}\left(L_{K}(E) p p^{*}, \bigoplus_{p \in Y} L_{K}(E) p p^{*}\right) \supseteq \prod_{p \in X} F_{p},
\end{aligned}
$$

where $F_{p} \cong K$. By Lemma 4.3, $\prod_{p \in X} F_{p}$ has $K$-dimension $2^{\sigma}$ and hence $H_{o m} o_{L_{K}(E)}(S, S)$ has $K$-dimension $\geq 2^{\sigma}$. On the other hand, $\operatorname{Hom}_{L_{K}(E)}\left(L_{K}(E) v, L_{K}(E) v\right) \cong v L_{K}(E) v$ has, as noted earlier, $K$-dimension $\leq \sigma$ and this contradicts the fact that $\phi^{*}$ is an epimorphism. Hence the graph $E$ must be row-finite.

Proposition 4.5. If a Leavitt path algebra $L_{K}(E)$ is left (right) self-injective and $a \in L_{K}(E)$, then $L_{K}(E) a\left(a L_{K}(E)\right)$ cannot have an infinite set of $L_{K}(E)$-independent left (right) ideals of $L_{K}(E)$.

Proof. Now by Proposition 4.4, the graph $E$ is row-finite. Let $a \in L_{K}(E)$ and, since $L_{K}(E)$ has local units, $L_{K}(E) a \subseteq L_{K}(E) \epsilon$ for some idempotent $\epsilon$. The proof that $L_{K}(E) \epsilon$ (and hence $\left.L_{K}(E) a\right)$ has finite uniform dimension is similar to that of Proposition 4.4. Suppose $L_{K}(E) \epsilon$ has an infinite family of independent submodules $\left\{A_{k} \mid k \in I\right\}$ and let $S=\bigoplus_{k \in I} A_{k}$ where $I$ is an infinite set. Write $\epsilon=\sum l_{j} a_{j} b_{j}^{*}$ where $a_{j}$ and $b_{j}$ are finite paths and $l_{j} \in K$. Let $v_{1}, \ldots, v_{m}$ be the vertices that appear as $s\left(a_{j}\right)$ or $s\left(b_{j}\right)$ of the finitely many paths $a_{j}$ and $b_{j}$. Then every element of $\epsilon L_{K}(E) \epsilon$ is of the form $\sum_{i=1}^{n} k_{i} p_{i} q_{i}^{*}$ where $s\left(p_{i}\right)=s\left(q_{i}\right) \in\left\{v_{1}, \ldots, v_{m}\right\}$. Since $E$ is row-finite, the cardinality of paths of a fixed length $n$ beginning with any of the vertices $v_{1}, \ldots, v_{m}$ is finite and hence the cardinality of the set of all paths of finite length beginning with any of these vertices $v_{1}, \ldots, v_{m}$ is at most countable. As in the proof of Proposition 4.4, we then conclude that the $K$-dimension of $\epsilon L_{K}(E) \epsilon$ is at most countable.

On the other hand, $\operatorname{Hom}_{L_{K}(E)}(S, S)=\operatorname{Hom}_{L_{K}(E)}\left(\bigoplus_{k \in I} A_{k}, \bigoplus_{k \in I} A_{k}\right) \supseteq \prod_{n=1}^{\infty} F_{n}$ where $F_{n} \cong K$ and so $H_{o m} m_{L_{K}(E)}(S, S)$ has $K$-dimension $\geq 2^{\aleph_{0}}$. Since the direct summand $L_{K}(E) \epsilon$ of $L_{K}(E)$ is an injective $L_{K}(E)$-module, the inclusion map $j: S \rightarrow L_{K}(E) \epsilon$ will induce an epimorphism $j^{*}: \operatorname{Hom}_{L_{K}(E)}\left(L_{K}(E) \epsilon, L_{K}(E) \epsilon\right) \rightarrow \operatorname{Hom}_{L_{K}(E)}(S, S)$ and this is not possible, since $\operatorname{Hom}_{L_{K}(E)}\left(L_{K}(E) \epsilon, L_{K}(E) \epsilon\right) \cong \epsilon L_{K}(E) \epsilon$ has countable $K$-dimension. Hence $L_{K}(E) \epsilon$ and so $L_{K}(E) a$ must have finite uniform dimension. Similar arguments apply when $L_{K}(E)$ is right self-injective.

The following graph definitions will be useful in the characterization of self-injective Leavitt path algebras in Theorem 4.7 .

Recall that a vertex $v$ in $E^{0}$ is a bifurcation (or that there is a bifurcation at $v$ ) if $s^{-1}(v)$ has at least two elements, and we say that there exists a cycle at $v$ if $v$ is a vertex of some cycle. A vertex $u$ in $E^{0}$ will be called a line point if there are neither bifurcations nor cycles at any vertex $w \in T(u)$. We will denote by $P_{l}(E)$ the set of all line points in $E^{0}$. Clearly $P_{l}(E)$ is always a hereditary set.

We say that an infinite path $\gamma=\left(e_{n}\right)_{n=1}^{\infty}$ ends in a sink if there exists $m \geq 1$ such that the infinite subpath $\mu=\left(e_{n}\right)_{n=m}^{\infty}$ has neither bifurcations nor cycles, or equivalently, if 
$\mu^{0} \subseteq P_{l}(E)$, i.e, the path $\mu$ contains a line point. In this situation the infinite path $\mu$ is called an infinite sink.

Proposition 4.6. For any graph $E$ and every field $K$, if the Leavitt path algebra $L_{K}(E)$ is left (right) self-injective, then every infinite path in $E$ must end in a sink.

Proof. Suppose, on the contrary, $E$ contains an infinite path $\gamma$ which does not end in a sink. We follow the ideas in the proof of [6, Theorem 2.4] to reach a contradiction. Now $E$ is acyclic by Proposition 4.1 and so $\gamma$ will contain infinitely many bifurcation vertices $\left\{v_{i} \mid i=1,2,3, \ldots\right\}$ and we can write $\gamma$ as a concatenation of countably many paths, $\gamma=\gamma_{1} \gamma_{2} \ldots \gamma_{n} \ldots$ where $r\left(\gamma_{n}\right)=v_{n}$ for all $n$. Let $s(\gamma)=v$. For each positive integer $n$, let $e_{n}=\gamma_{1} \gamma_{2} \ldots \gamma_{n} \gamma_{n}^{*} \ldots \gamma_{2}^{*} \gamma_{1}^{*} v$. Observe that $e_{n+1}=e_{n+1} e_{n}$ for all $n$. But $e_{n} \neq r e_{n+1}$ for any $r \in L_{K}(E)$. Indeed, suppose $e_{n}=r e_{n+1}$ for some $r \in L_{K}(E)$. Since $v_{n}$ is a bifurcation vertex, there is an edge $f_{n}$ which is not the initial edge of $\gamma_{n+1}$ with $s\left(f_{n}\right)=s\left(\gamma_{n+1}\right)=v_{n}$. Clearly $\gamma_{n+1}^{*} f_{n}=0$. Then multiplying $e_{n}=r e_{n+1}$ on the right side by $\gamma_{1} \gamma_{2} \ldots \gamma_{n} f_{n}$, we obtain,

$$
0 \neq \gamma_{1} \gamma_{2} \ldots \gamma_{n} f_{n}=e_{n} \gamma_{1} \gamma_{2} \ldots \gamma_{n} f_{n}=r e_{n+1} \gamma_{1} \gamma_{2} \ldots \gamma_{n} f_{n}=r \gamma_{1} \gamma_{2} \ldots \gamma_{n+1} \gamma_{n+1}^{*} f_{n}=0,
$$

a contradiction. In particular, we conclude that $e_{n}-e_{n+1} \neq 0$ for all $n \geq 1$.

It is easy to check that the set $\left\{e_{n}-e_{n+1} \mid n=1,2,3, \ldots\right\}$ is an infinite set of nonzero mutually orthogonal idempotent elements in $L_{K}(E) v$ and so $\left\{L_{K}(E)\left(e_{n}-e_{n+1}\right) \mid n=\right.$ $1,2,3, \ldots\}$ is a countably infinite independent family of left ideals in $L_{K}(E) v$. By Proposition 4.5 , this is a contradiction.

We are now ready to prove the main theorem of this section.

Theorem 4.7. Let $E$ be an arbitrary graph and $K$ be any field. Then the following conditions are equivalent:

(i) $L_{K}(E)$ is left self-injective.

(ii) $E$ is row-finite, acyclic and every infinite path contains a line point.

(iii) $L_{K}(E)$ is right self-injective.

(iv) $L_{K}(E)$ is semisimple.

Proof. (i) $\Rightarrow$ (ii) follows from Propositions 4.1, 4.4 and 4.6.

(ii) $\Rightarrow$ (iv). This is proved in [6, Theorem 2.4].

(iv) $\Rightarrow$ (i). Since $L_{K}(E)$ is a direct sum of simple left ideals, then every unital left $L_{K}(E)$ module $M$ is also a direct sum of simple $L_{K}(E)$-submodules. Since every submodule of a semisimple module is a direct summand, every $L_{K}(E)$-module and, in particular $L_{K}(E)$, is injective as a left $L_{K}(E)$-module.

(iii) $\Leftrightarrow$ (iv) is proved in a similar fashion to that of (i) $\Leftrightarrow$ (iv) because the condition for being semisimple is left-right symmetric for semiprime rings, so in particular for $L_{K}(E)$ by [22, Proposition 3.4].

\section{ACKNOWLEDGMENTS}

This work was done when the second author visited the University of Málaga during MayJune 2009 and he gratefully acknowledges the kind hospitality of the faculty in the Algebra, Geometry and Topology Department. The first and third authors were partially supported by the Spanish MEC and Fondos FEDER through project MTM2007-60333, and by the Junta de Andalucía and Fondos FEDER, jointly, through projects FQM-336 and FQM-2467. This 
work has also been supported by the Spanish Ministry of Education and Science under project "Ingenio Mathematica (i-math)" No. CSD2006-00032 (Consolider-Ingenio 2010).

\section{REFERENCES}

[1] G. Abrams, P. N. Ánh, A. Louly, E. Pardo, The classification question for Leavitt algebras, J. Algebra 320 (2008), 1983-2026.

[2] G. Abrams, A. Louly, E. Pardo, C. Smith, Flow invariants in the classification of Leavitt path algebras, Preprint (2008).

[3] G. Abrams, G. Aranda Pino, The Leavitt path algebra of a graph, J. Algebra 293 (2) (2005), 319-334.

[4] G. Abrams, G. Aranda Pino, Purely infinite simple Leavitt path algebras, J. Pure Appl. Algebra 207 (3) (2006), 553-563.

[5] G. Abrams, G. Aranda Pino, The Leavitt path algebras of arbitrary graphs, Houston J. Math. 34 (2) (2008), 423-442.

[6] G. Abrams, G. Aranda Pino, F. Perera, M. Siles Molina, Chain conditions for Leavitt path algebras. Forum Math. (To appear).

[7] G. Abrams and K.M. Rangaswamy, Regularity conditions for arbitrary Leavitt path algebras, Algebr. Represent. Theory. (To appear).

[8] G. Abrams, K.M. Rangaswamy, M. Siles Molina, The socle series of a Leavitt path algebra. Israel J. Math. (To appear).

[9] G. Abrams, M. Tomforde, Isomorphism and Morita equivalence of graph algebras, (preprint).

[10] P. Alberca Bjerregandd, G. Aranda Pino, D. Martín Barquero, C. Martín González, M. Siles Molina, Atlas for Leavitt path algebras of small graphs, (in preparation).

[11] R. R. Andruszkiewicz, E. R. Puczylowski, Right fully idempotent rings need not be left fully idempotent, Glasgow Math. J. 37 (1995), 155-157.

[12] P. N. ÁnH, L. MÁRKI, Left orders in regular rings with minimum condition for principal one-sided ideals, Math. Proc. Phil. Soc. 109 (1991), 323-333.

[13] P. N. Ánh, L. MÁrki, Morita equivalence for rings without identity, Tsukuba J. Math. 11 (1) (1987), $1-16$.

[14] P. Ara, M. Brustenga, Module theory over Leavitt path algebras and K-theory, (Preprint) arXiv:0905.3827v1.

[15] P. Ara, M. Brustenga, G. Cortiñas, K-theory for Leavitt path algebras, Münster J. of Math (To appear.) arXiv:0903.0056.

[16] P. Ara, M. Gómez Lozano, M. Siles Molina, Local rings of exchange rings Comm. in Algebra 26(12) (1998), 4191-4205.

[17] P. Ara, M.A. Moreno, E. Pardo, Nonstable K-Theory for graph algebras, Algebra Represent. Theory 10 (2007), 157-178.

[18] P. Ara, E. Pardo, Stable rank for graph algebras, Proc. Amer. Math. Soc. 136 (7) (2008), 2375-2386.

[19] G. Aranda Pino, K. Crow, The center of a Leavitt path algebra, (Preprint).

[20] G. Aranda Pino, K. R. Goodearl, F. Perera, M. Siles Molina, Non-simple purely infinite rings, Amer. J. Math., (to appear).

[21] G. Aranda Pino, D. Martín Barquero, C. Martín GonzÁlez, M. Siles Molina, The socle of a Leavitt path algebra, J. Pure Appl. Algebra 212 (3) (2008), 500-509.

[22] G. Aranda Pino, D. Martín Barquero, C. Martín González, M. Siles Molina, Socle theory for Leavitt path algebras of arbitrary graphs, Rev. Mat Iber. (To appear).

[23] G. Aranda Pino, E. Pardo, M. Siles Molina, Exchange Leavitt path algebras and stable rank, J. Algebra 305 (2) (2006), 912-936.

[24] G. Aranda Pino, E. Pardo, M. Siles Molina, Prime spectrum and primitive Leavitt path algebras, Indiana Univ. Math. J. 58 (2) (2009), 869-890.

[25] G. Aranda Pino, F. Perera, M. Siles Molina, eds., Graph algebras: bridging the gap between analysis and algebra, ISBN: 978-84-9747-177-0, University of Málaga Press, Málaga, Spain (2007). 
[26] K. I. Beidar, W. S. Martindale 3Rd, A. V. Mikhalev, Rings with generalized identities, Marcel Dekker, 1996.

[27] J. Cuntz, Simple $C^{*}$-algebras generated by isometries, Comm. Math. Phys. 57 (1977), 173-185.

[28] K. Deicke, J.H. Hong, W. Szymański, Stable rank of graph algebras. Type I graph algebras and their limits, Indiana Univ. Math. J. 52(4) (2003), 963-979.

[29] C. FAith, Lectures on Injective Modules and Quotient Rings, Lecture Notes in Math., vol. 49, SpringerVerlag (1967).

[30] L. Fuchs, Infinite Abelian groups, vol. I, Pure and Applied Mathematics, Vol. 36 Academic Press, New York-London (1970).

[31] J. L. García, J.J. Simón, Morita equivalence for idempotent rings. J. Pure Appl. Algebra 76 (1991), $39-56$.

[32] K. R. Goodearl, Leavitt path algebras and direct limits, Contemp. Math. 480 (200), 165-187.

[33] C. Y. Hong, N. K. Kim, Y. Lee, Exchange rings and their extensions. J. Pure Appl. Algebra 179 (2003), 117-126.

[34] N. Jacobson, Structure of Rings, Amer. Math. Soc. Colloquium Publications, vol. 37 (1964).

[35] T. Jech, Set Theory, Springer Monographs in Mathematics, Springer-Verlag, NY (1997).

[36] S. Kyuno, Equivalence of module categories. Math. J. Okayama Univ. 28 (1974), 147-150.

[37] T.Y. LaM, Lectures on Modules and Rings, Springer-Verlag New York (1999).

[38] W. G. Leavitt, Modules without invariant basis number, Proc. Amer. Math. Soc. 8 (1957), 322-328.

[39] I. Raeburn, Graph algebras. CBMS Regional Conference Series in Mathematics, 103, Amer. Math. Soc., Providence (2005).

[40] V. S. Ramamurthi, Weakly regular rings, Canad. Math. Bull. 16 (1973), 317-321.

[41] J. Rotman, An introduction to homological algebra. Second edition, Universitext. Springer, New York (2009).

[42] M. Siles Molina, Algebras of quotients of path algebras, J. Algebra 319 (12) (2008), 329-348.

[43] M. Tomforde, Uniqueness theorems and ideal structure for Leavitt path algebras, J. Algebra 318 (1) (2007), 270-299.

[44] A. Tuganbaev, Rings close to regular, Mathematics and its Applications, 545. Kluwer Academic Publishers, Dordrecht (2002).

Aranda and Siles: Departamento de Álgebra, Geometría y Topología, Universidad de MÁlaga, 29071 Málaga, SpAin

E-mail address: g.aranda@uma.es, msilesm@uma.es

Rangaswamy: Department of Mathematics, University of Colorado, Colorado Springs CO 80933 U.S.A.

E-mail address: ranga@uccs.edu 